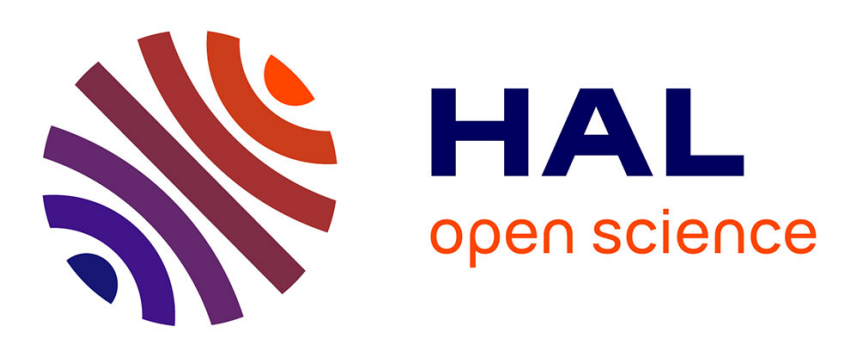

\title{
Discontinuous Galerkin gradient discretisations for the approximation of second-order differential operators in divergence form
}

\author{
Robert Eymard, Cindy Guichard
}

\section{- To cite this version:}

Robert Eymard, Cindy Guichard. Discontinuous Galerkin gradient discretisations for the approximation of second-order differential operators in divergence form. Computational \& Applied Mathematics, 2018, 37 (4), pp.:4023-4054. 10.1007/s40314-017-0558-2 . hal-01535147v2

HAL Id: hal-01535147

https://hal.science/hal-01535147v2

Submitted on 20 Nov 2017

HAL is a multi-disciplinary open access archive for the deposit and dissemination of scientific research documents, whether they are published or not. The documents may come from teaching and research institutions in France or abroad, or from public or private research centers.
L'archive ouverte pluridisciplinaire HAL, est destinée au dépôt et à la diffusion de documents scientifiques de niveau recherche, publiés ou non, émanant des établissements d'enseignement et de recherche français ou étrangers, des laboratoires publics ou privés. 


\title{
Discontinuous Galerkin gradient discretisations for the approximation of second-order differential operators in divergence form
}

\author{
Robert Eymard*and Cindy Guichard ${ }^{\dagger}$
}

\begin{abstract}
We include in the Gradient Discretisation Method (GDM) framework two numerical schemes based on Discontinuous Galerkin approximations: the Symmetric Interior Penalty Galerkin (SIPG) method, and the scheme obtained by averaging the jumps in the SIPG method. We prove that these schemes meet the main mathematical gradient discretisation properties on any kind of polytopal mesh, by adapting discrete functional analysis properties to our precise geometrical hypotheses. Therefore, these schemes inherit the general convergence properties of the GDM, which hold for instance in the cases of the $p$-Laplace problem and of the anisotropic and heterogeneous diffusion problem. This is illustrated by simple $1 \mathrm{D}$ and $2 \mathrm{D}$ numerical examples.

Keywords: Gradient Discretisation method, Discontinuous Galerkin method, Symmetric Interior Penalty
\end{abstract} Galerkin scheme, discrete functional analysis, polytopal meshes

\section{Introduction}

Discontinuous Galerkin (DG) methods have received a lot of attention over the last decade at least, and are still a subject of interest. They present the advantage to be suited to elliptic and parabolic problems, while opening the possibility to closely approximate weakly regular functions on general meshes. Although the convergence of DG methods has been proved on a variety of problems (see [8] and references therein), note that the stabilisation of DG schemes for elliptic or parabolic problems has to be specified with respect to the problem, and that there are numerous possible choices [2].

On the other hand, convergence and error estimate results for a wide variety of numerical methods applied to some elliptic, parabolic, coupled, linear and nonlinear problems are proved on the generic "gradient scheme" issued from the Gradient Discretisation Method framework (see [9] and references therein). This framework is shown to include conforming Galerkin methods with or without mass lumping, nonconforming $\mathbb{P}^{1}$ finite elements, mixed finite elements and a variety of schemes issued from extensions of the finite volume method. Convergence and error estimate results are then proved in [9] for the following problems:

1. elliptic problem with constant or unknown dependent diffusion,

2. steady or transient $p$-Laplace problem and more generally Leray-Lions problem,

3. parabolic problem with constant or unknown dependent diffusion,

4. degenerate parabolic (Richards or Stefan-type) problems,

provided that the Gradient Discretisation is coercive, GD-consistent, limit-conforming, compact and in some cases with piecewise constant reconstruction (the precise mathematical meaning of these core properties is recalled in Section 2 of this paper).

The aim of this paper is to build Gradient Discretisations from the DG setting. Considering two ways of accounting for the jump terms (pointwise or by mean value), we define on one hand the Discontinuous Galerkin Gradient Discretisation (DGGD) and on the other hand the Average Discontinuous Galerkin Gradient Discretisation (ADGGD). In both cases, we show that all the core properties of GDM are satisfied on general polytopal meshes in any space dimension. This immediately extends the range of problems which 
can be handled by Discontinuous Galerkin methods to all the above problems, as we show in this paper by considering the case of the $p$-Laplace problem. Indeed, we can immediately apply a convergence and error estimate result derived from the Gradient Discretisation Method framework. This approach is different from that of [4] where a specific stabilisation term is introduced in the variational formulation, whereas the present work has significant common points with the stable DG method without penalty parameter proposed in [15] (where a scheme, which can enter into the GDM, is proposed, the main difference with the present paper being the lifting of the jumps for computing the discrete gradient).

It is then interesting to remark that the gradient scheme resulting from the DGGD is identical to the Symmetric Interior Penalty Galerkin scheme in the case of linear diffusion problems, as we detail in section 6. Note that the gradient scheme resulting from the ADGGD is not identical, in the case of the elasticity problem, to the scheme introduced in [13], in which the volumetric part is handled with average jumps but the deviatoric part is handled with pointwise jumps.

This paper is organised as follows. Section 2 includes the main definitions for the Gradient Discretisation Method in the case of homogeneous Dirichlet boundary conditions. In Section 3, we study a gradient discretisation version of Discontinuous Galerkin schemes suited to polytopal meshes in any space dimension using pointwise jumps, and we prove that this gradient discretisation satisfies the core properties which are sufficient for convergence and error estimates results. We turn in Section 4 to the case of average jumps, that is studied owing to a result of equivalence of norms between the DG norm build with pointwise jumps and that build with average jumps. We then take an example of application of the preceding results in Section 5, where the gradient schemes issued from the DGGD and ADGGD methods are shown to satisfy a convergence and error estimate in the case of the $p$-Laplace problem. We then handle in Section 6 the case of linear elliptic problems, and we show that the scheme issued from the DGGD is identical to the Symmetric Interior Penalty Galerkin method. A numerical example shows the role the numerical parameter used in the design of the scheme plays in its accuracy. A short conclusion is then proposed, before the adaptation of the Sobolev inequalities proved in $[7,8]$ to our setting and definition of the DG norm in Appendix A. Note that the assumption that the mesh cells are star-shaped with respect to some point (not made in $[7,8]$ ) enables to prove that the constant involved in these inequalities do not depend on the regularity factor of the mesh.

\section{Main definitions of Gradient Discretisations for homogeneous Dirichlet boundary conditions}

In this paper, we make the following assumptions: $p \in(1,+\infty)$ is given and

$$
\Omega \text { is an open bounded polytopal connected subset of } \mathbb{R}^{d}\left(d \in \mathbb{N}^{\star}\right),
$$

where polytopal means that it is the union of a finite number of simplices.

The following definitions, first introduced in [12], are given in [9] for a larger variety of boundary conditions.

Definition 2.1 (GD, homogeneous Dirichlet BCs):

A gradient discretisation $\mathcal{D}$ for homogeneous Dirichlet conditions is defined by $\mathcal{D}=\left(X_{\mathcal{D}, 0}, \Pi_{\mathcal{D}}, \nabla_{\mathcal{D}}\right)$, where:

1. the set of discrete unknowns $X_{\mathcal{D}, 0}$ is a finite dimensional real vector space,

2. the function reconstruction $\Pi_{\mathcal{D}}: X_{\mathcal{D}, 0} \rightarrow L^{p}(\Omega)$ is a linear mapping that reconstructs, from an element of $X_{\mathcal{D}, 0}$, a function over $\Omega$,

3. the gradient reconstruction $\nabla_{\mathcal{D}}: X_{\mathcal{D}, 0} \rightarrow L^{p}(\Omega)^{d}$ is a linear mapping which reconstructs, from an element of $X_{\mathcal{D}, 0}$, a "gradient" (vector-valued function) over $\Omega$. This gradient reconstruction must be chosen such that $\left\|\nabla_{\mathcal{D}} \cdot\right\|_{L^{p}(\Omega)^{d}}$ is a norm on $X_{\mathcal{D}, 0}$.

Definition 2.2 (Coercivity): If $\mathcal{D}$ is a gradient discretisation in the sense of Definition 2.1, define $C_{\mathcal{D}}$ as the norm of the linear mapping $\Pi_{\mathcal{D}}$ :

$$
C_{\mathcal{D}}=\max _{v \in X_{\mathcal{D}, 0} \backslash\{0\}} \frac{\left\|\Pi_{\mathcal{D}} v\right\|_{L^{p}(\Omega)}}{\left\|\nabla_{\mathcal{D}} v\right\|_{L^{p}(\Omega)^{d}}} .
$$

A sequence $\left(\mathcal{D}_{m}\right)_{m \in \mathbb{N}}$ of gradient discretisations in the sense of Definition 2.1 is coercive if there exists $C_{P} \in \mathbb{R}_{+}$such that $C_{\mathcal{D}_{m}} \leq C_{P}$ for all $m \in \mathbb{N}$. 
The consistency properties indicate how accurately a regular function and its gradient are approximated by some discrete function and gradient which are reconstructed from the space $X_{\mathcal{D}, 0}$.

Definition 2.3 (GD-consistency): If $\mathcal{D}$ is a gradient discretisation in the sense of Definition 2.1, define $S_{\mathcal{D}}$ : $W_{0}^{1, p}(\Omega) \rightarrow[0,+\infty)$ by

$$
\forall \varphi \in W_{0}^{1, p}(\Omega), S_{\mathcal{D}}(\varphi)=\min _{v \in X_{\mathcal{D}, 0}}\left(\left\|\Pi_{\mathcal{D}} v-\varphi\right\|_{L^{p}(\Omega)}+\left\|\nabla_{\mathcal{D}} v-\nabla \varphi\right\|_{L^{p}(\Omega)^{d}}\right) .
$$

A sequence $\left(\mathcal{D}_{m}\right)_{m \in \mathbb{N}}$ of gradient discretisations in the sense of Definition 2.1 is GD-consistent, or consistent for short, if

$$
\forall \varphi \in W_{0}^{1, p}(\Omega), \lim _{m \rightarrow \infty} S_{\mathcal{D}_{m}}(\varphi)=0
$$

The concept of limit-conformity which we now introduce states that the discrete gradient and divergence operator satisfy this property asymptotically. Since we shall be dealing with non linear problems, we introduce, or any $q \in(1,+\infty)$, the space $W_{\text {div }}^{q}(\Omega)$ of functions in $\left(L^{q}(\Omega)\right)^{d}$ with divergence in $L^{q}(\Omega)$ :

$$
W_{\mathrm{div}}^{q}(\Omega)=\left\{\varphi \in L^{q}(\Omega)^{d}: \operatorname{div} \varphi \in L^{q}(\Omega)\right\} .
$$

Definition 2.4 (Limit-conformity): If $\mathcal{D}$ is a gradient discretisation in the sense of Definition 2.1 , let $p^{\prime}=\frac{p}{p-1}$ and define $W_{\mathcal{D}}: W_{\text {div }}^{p^{\prime}}(\Omega) \rightarrow[0,+\infty)$ by

$$
\forall \boldsymbol{\varphi} \in W_{\mathrm{div}}^{p^{\prime}}(\Omega), W_{\mathcal{D}}(\boldsymbol{\varphi})=\sup _{v \in X_{\mathcal{D}, 0 \backslash\{0\}}} \frac{\left|\int_{\Omega}\left(\nabla_{\mathcal{D}} v(\boldsymbol{x}) \cdot \boldsymbol{\varphi}(\boldsymbol{x})+\Pi_{\mathcal{D}} v(\boldsymbol{x}) \operatorname{div} \boldsymbol{\varphi}(\boldsymbol{x})\right) \mathrm{d} \boldsymbol{x}\right|}{\left\|\nabla_{\mathcal{D}} v\right\|_{L^{p}(\Omega)^{d}}} .
$$

A sequence $\left(\mathcal{D}_{m}\right)_{m \in \mathbb{N}}$ of gradient discretisations is limit-conforming if

$$
\forall \boldsymbol{\varphi} \in W_{\mathrm{div}}^{p^{\prime}}(\Omega), \lim _{m \rightarrow \infty} W_{\mathcal{D}_{m}}(\boldsymbol{\varphi})=0 .
$$

Dealing with generic non-linearity often requires additional compactness properties on the scheme.

Definition 2.5 (Compactness): A sequence $\left(\mathcal{D}_{m}\right)_{m \in \mathbb{N}}$ of gradient discretisations in the sense of Definition 2.1 is compact if, for any sequence $u_{m} \in X_{\mathcal{D}_{m}, 0}$ such that $\left(\left\|\nabla_{\mathcal{D}_{m}} u_{m}\right\|_{L^{p}(\Omega)}\right)_{m \in \mathbb{N}}$ is bounded, the sequence $\left(\Pi_{\mathcal{D}_{m}} u_{m}\right)_{m \in \mathbb{N}}$ is relatively compact in $L^{p}(\Omega)$.

Definition 2.6 (Piecewise constant reconstruction): Let $\mathcal{D}=\left(X_{\mathcal{D}, 0}, \Pi_{\mathcal{D}}, \nabla_{\mathcal{D}}\right)$ be a gradient discretisation in the sense of Definition 2.1. The operator $\Pi_{\mathcal{D}}: X_{\mathcal{D}, 0} \rightarrow L^{p}(\Omega)$ is a piecewise constant reconstruction if there exists a basis $\left(e_{i}\right)_{i \in B}$ of $X_{\mathcal{D}, 0}$ and a family of disjoint subsets $\left(\Omega_{i}\right)_{i \in B}$ of $\Omega$ such that $\Pi_{\mathcal{D}} e_{i}=\mathbf{1}_{\Omega_{i}}$ for all $i \in B$, where $\mathbf{1}_{\Omega_{i}}$ is the characteristic function of $\Omega_{i}$.

It is shown in [9] that all the considered examples of Gradient Discretisations (as listed in the introduction of this paper) meet four of the core properties (coercivity, GD-consistency, limit-conformity, compactness), and that mass-lumped versions satisfy the piece-wise constant reconstruction property. They therefore satisfy convergence and error estimates for the range of problems passed into review in the introduction of this paper. The next sections aim to build a Gradient Discretisation with the discontinuous Galerkin setting, and then to prove that it satisfies the core properties as well.

\section{Discontinuous Galerkin Gradient Discretisation (DGGD)}

\subsection{Meshes and discrete operators}

Let us provide a definition for a polytopal mesh of $\Omega$, which is a slightly simplified version of that given in $[9]$.

Definition 3.1 (Polytopal mesh): A polytopal mesh of $\Omega$ is given by $\mathfrak{T}=(\mathcal{M}, \mathcal{F}, \mathcal{P})$, where: 
1. $\mathcal{M}$ is a finite family of non empty connected polytopal open disjoint subsets of $\Omega$ (the "cells") such that $\bar{\Omega}=\cup_{K \in \mathcal{M}} \bar{K}$. For any $K \in \mathcal{M}$, let $\partial K=\bar{K} \backslash K$ be the boundary of $K,|K|>0$ is the measure of $K$ and $h_{K}$ denotes the diameter of $K$, that is the maximum distance between two points of $\bar{K}$.

2. $\mathcal{F}=\mathcal{F}_{\text {int }} \cup \mathcal{F}_{\text {ext }}$ is a finite family of disjoint subsets of $\bar{\Omega}$ (the "faces" of the mesh - "edges" in $2 \mathrm{D}$ ), such that, for all $\sigma \in \mathcal{F}_{\text {int }}, \sigma$ is a non empty open subset of a hyperplane of $\mathbb{R}^{d}$ included in $\Omega$ and, for all $\sigma \in \mathcal{F}_{\text {ext }}, \sigma$ is a non empty open subset of $\partial \Omega$; furthermore, the $(d-1)$-dimensional measure $|\sigma|$ of any $\sigma \in \mathcal{F}$ is strictly positive. We assume that, for all $K \in \mathcal{M}$, there exists a subset $\mathcal{F}_{K}$ of $\mathcal{F}$ such that $\partial K=\cup_{\sigma \in \mathcal{F}_{K}} \bar{\sigma}$. We then denote by $\mathcal{M}_{\sigma}=\left\{K \in \mathcal{M}, \sigma \in \mathcal{F}_{K}\right\}$. We then assume that, for all $\sigma \in \mathcal{F}$, either $\mathcal{M}_{\sigma}$ has exactly one element and then $\sigma \in \mathcal{F}_{\text {ext }}$ or $\mathcal{M}_{\sigma}$ has exactly two elements and then $\sigma \in \mathcal{F}_{\text {int. }}$. For all $K \in \mathcal{M}$ and for any $\sigma \in \mathcal{F}_{K}$, we denote by $\boldsymbol{n}_{K, \sigma}$ the (constant) unit vector normal to $\sigma$ outward to $K$.

3. $\mathcal{P}$ is a family of points of $\Omega$ indexed by $\mathcal{M}$, denoted by $\mathcal{P}=\left(\boldsymbol{x}_{K}\right)_{K \in \mathcal{M}}$, such that for all $K \in \mathcal{M}$, $\boldsymbol{x}_{K} \in K$. We then denote by $d_{K, \sigma}$ the signed orthogonal distance between $\boldsymbol{x}_{K}$ and $\sigma \in \mathcal{F}_{K}$ (see Figure 1), that is:

$$
d_{K, \sigma}=\left(\boldsymbol{x}-\boldsymbol{x}_{K}\right) \cdot \boldsymbol{n}_{K, \sigma}, \text { for all } \boldsymbol{x} \in \sigma .
$$

We then assume that each cell $K \in \mathcal{M}$ is strictly star-shaped with respect to $\boldsymbol{x}_{K}$, that is $d_{K, \sigma}>0$ for all $\sigma \in \mathcal{F}_{K}$. This implies that for all $\boldsymbol{x} \in K$, the line segment $\left[\boldsymbol{x}_{K}, \boldsymbol{x}\right]$ is included in $K$.

For all $K \in \mathcal{M}$ and $\sigma \in \mathcal{F}_{K}$, we denote by $D_{K, \sigma}$ the cone with vertex $\boldsymbol{x}_{K}$ and basis $\sigma$, that is

$$
D_{K, \sigma}=\left\{\boldsymbol{x}_{K}+s\left(\boldsymbol{y}-\boldsymbol{x}_{K}\right), s \in(0,1), \boldsymbol{y} \in \sigma\right\} .
$$

The size of the polytopal mesh is defined by:

$$
h_{\mathcal{M}}=\sup \left\{h_{K}, K \in \mathcal{M}\right\} .
$$

Finally, for a given polytopal mesh $\mathfrak{T}$ we define a number that measures the regularity properties of the mesh:

$$
\eta_{\mathfrak{T}}=\max \left(\left\{\frac{h_{K}}{h_{L}}+\frac{h_{L}}{h_{K}}, \sigma \in \mathcal{F}_{\mathrm{int}}, \mathcal{M}_{\sigma}=\{K, L\}\right\} \cup\left\{\frac{h_{K}}{d_{K, \sigma}}, K \in \mathcal{M}, \sigma \in \mathcal{F}_{K}\right\} \cup\left\{\# \mathcal{F}_{K}, K \in \mathcal{M}\right\}\right),
$$

where we denote by $\# \mathcal{F}_{K}$ the cardinal of the set $\mathcal{F}_{K}$.
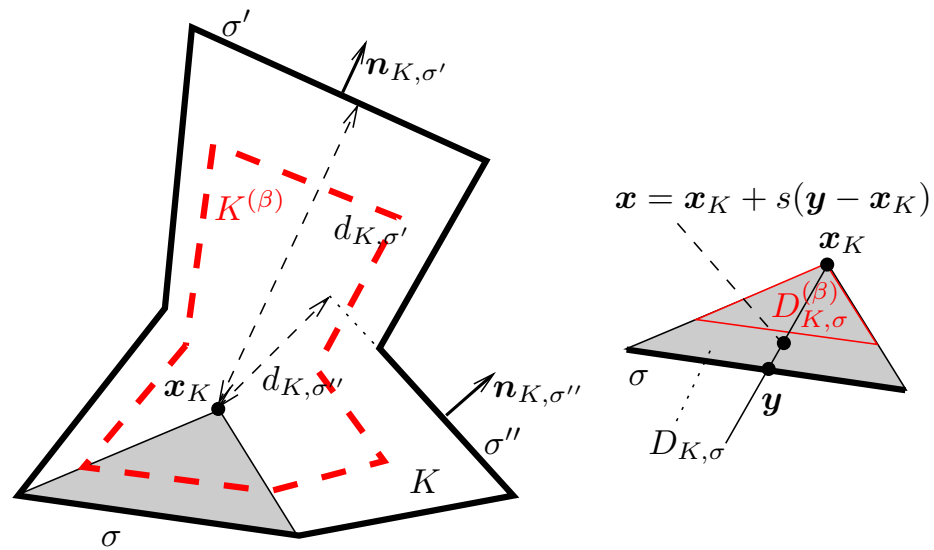

Fig. 1: A cell $K$ of a polytopal mesh and notation on $D_{K, \sigma}$

Let us now define, using the discontinuous Galerkin framework, a gradient discretisation in the sense of Definition 2.1. 


\section{Definition 3.2: [Discontinuous Galerkin Gradient Discretisation}

(DGGD)] Let $\mathfrak{T}=(\mathcal{M}, \mathcal{F}, \mathcal{P})$ be a polytopal mesh of $\Omega$ in the sense of Definition 3.1. Define the Discontinuous Galerkin Gradient Discretisation $\mathcal{D}=\left(X_{\mathcal{D}, 0}, \Pi_{\mathcal{D}}, \nabla_{\mathcal{D}}\right)$ by the following.

1. For a given value $k \in \mathbb{N}^{\star}$, we consider the space $W_{h}$, defined by

$$
W_{h}=\left\{u \in L^{p}(\Omega):\left.u\right|_{K} \in \mathbb{P}_{k}(K), \forall K \in \mathcal{M}\right\} .
$$

Recall that the dimension of $\mathbb{P}_{k}\left(\mathbb{R}^{d}\right)$ is $\frac{(k+d) !}{k ! d !}$, and therefore the dimension of $W_{h}$ is equal to $\frac{(k+d) !}{k ! d !} \# \mathcal{M}$. Let $\left(\chi_{i}\right)_{i \in I}$ be a family of piecewise polynomial basis functions of degree $k$ on each cell, spanning $W_{h}$. We set

$$
X_{\mathcal{D}, 0}=\left\{v=\left(v_{i}\right)_{i \in I}: v_{i} \in \mathbb{R} \text { for all } i \in I\right\} .
$$

2. The operator $\Pi_{\mathcal{D}}$ is the reconstruction in $L^{p}(\Omega)$ of the elements of $X_{\mathcal{D}, 0}$ :

$$
\forall v \in X_{\mathcal{D}, 0}, \Pi_{\mathcal{D}} v=\sum_{i \in I} v_{i} \chi_{i}
$$

In this paper, we denote, for all $K \in \mathcal{M}$ and $v \in X_{\mathcal{D}, 0}$, by $\Pi_{\bar{K}} v \in \mathbb{P}_{k}(\bar{K})$ the piecewise polynomial defined by $\Pi_{\mathcal{D}} v$ on $K$, extended on $\bar{K}$, and we denote by $\nabla_{\bar{K}} v=\nabla \Pi_{\bar{K}} v$.

3. For $v \in X_{\mathcal{D}, 0}$, for $K \in \mathcal{M}$ and for any $\sigma \in \mathcal{F}_{K}$, we set, for a.e. $\boldsymbol{x} \in D_{K, \sigma}$,

$$
\nabla_{\mathcal{D}} v(\boldsymbol{x})=\nabla_{\bar{K}} v(\boldsymbol{x})+\psi(S(\boldsymbol{x})) \frac{[v]_{K, \sigma}(\boldsymbol{Y}(\boldsymbol{x}))}{d_{K, \sigma}} \boldsymbol{n}_{K, \sigma},
$$

where (see Figure 1):

- we denote by $S, \boldsymbol{Y}$ the functions $S: D_{K, \sigma} \rightarrow(0,1]$ and $\boldsymbol{Y}: D_{K, \sigma} \rightarrow \sigma$ such that $\boldsymbol{x}=$ $\boldsymbol{x}_{K}+S(\boldsymbol{x})\left(\boldsymbol{Y}(\boldsymbol{x})-\boldsymbol{x}_{K}\right)$, which means

$$
S(\boldsymbol{x})=\frac{\left(\boldsymbol{x}-\boldsymbol{x}_{K}\right) \cdot \boldsymbol{n}_{K, \sigma}}{d_{K, \sigma}} \text { and } \boldsymbol{Y}(\boldsymbol{x})=\boldsymbol{x}_{K}+\frac{\boldsymbol{x}-\boldsymbol{x}_{K}}{S(\boldsymbol{x})},
$$

- for all $K \in \mathcal{M}$ and all $\sigma \in \mathcal{F}_{K}$, we denote by

$$
\begin{gathered}
\forall \boldsymbol{y} \in \sigma, \quad \text { if } \mathcal{M}_{\sigma}=\{K, L\},[v]_{K, \sigma}(\boldsymbol{y})=\frac{1}{2}\left(\Pi_{\bar{L}} v(\boldsymbol{y})-\Pi_{\bar{K}} v(\boldsymbol{y})\right) \\
\text { else if } \mathcal{M}_{\sigma}=\{K\},[v]_{K, \sigma}(\boldsymbol{y})=0-\Pi_{\bar{K}} v(\boldsymbol{y}),
\end{gathered}
$$

- Let $\beta \in(0,1)$ be given. Let $\psi:(0,1) \rightarrow \mathbb{R}$ be the function such that $\psi(s)=0$ on $(0, \beta)$, $\psi_{\mid[\beta, 1]} \in \mathbb{P}_{k-1}([\beta, 1])$ and

$$
\begin{aligned}
& \int_{\beta}^{1} \psi(s) s^{d-1} \mathrm{~d} s=1 \\
& \forall i=1, \ldots, k-1, \int_{\beta}^{1}(1-s)^{i} \psi(s) s^{d-1} \mathrm{~d} s=0 .
\end{aligned}
$$

Remark 3.3: Assumption (17) is mandatory in the proof of the limit-conformity of the scheme (see (34) in Lemma 3.15), whereas (18) is used in the same equation for the high-order accuracy. The cut-off parameter $\beta \in(0,1)$ is necessary for getting the equivalence $(20)$ between the norm of the discrete gradient and the Discontinuous Galerkin norm (defined by (21)). Note that $\beta=0$ is considered in the numerical examples, but the present convergence analysis is no longer available in this case. Lower and upper bounds of $\|\psi\|_{\infty}$ are provided by (57) in Lemma A.1. 
In the case $k=1$, the function $\psi_{\mid[\beta, 1]} \in \mathbb{P}_{0}([\beta, 1])$ has the constant value $\frac{d}{1-\beta^{d}}$.

For the general case $k \in \mathbb{N}^{\star}$, considering the basis $\left(1,(1-s), \ldots,(1-s)^{k-1}\right)$ of $\mathbb{P}_{k-1}([\beta, 1])$, and writing the function $\psi$ as $\psi(s)=\sum_{i=1}^{k} \alpha_{i}(1-s)^{i-1}$, we see that the matrix $A$ of the linear system issued from (17)-(18) (it is a Gram matrix), with unknowns $\left(\alpha_{i}\right)_{i=1, \ldots, k}$, is such that

$$
A_{i, j}=\int_{\beta}^{1}(1-s)^{i+j-2} s^{d-1} \mathrm{~d} s .
$$

Considering a vector $U$ such that $U^{t}=\left(u_{i}\right)_{i=1, \ldots, k}$, we note that

$$
U^{t} A U=\int_{\beta}^{1}\left(\sum_{i=1}^{k} u_{i}(1-s)^{i-1}\right)^{2} s^{d-1} \mathrm{~d} s
$$

which implies that $A$ is symmetric definite positive, hence leading to the existence and uniqueness of $\psi$. We split $D_{K, \sigma}$ into $D_{K, \sigma}^{(\beta)}$ and $D_{K, \sigma} \backslash D_{K, \sigma}^{(\beta)}$ with

$$
\begin{aligned}
& D_{K, \sigma}^{(\beta)}:=\left\{\boldsymbol{x} \in D_{K, \sigma}, \boldsymbol{x}=\boldsymbol{x}_{K}+s\left(\boldsymbol{y}-\boldsymbol{x}_{K}\right), s \in(0, \beta], \boldsymbol{y} \in \sigma\right\} \\
& \text { and } K^{(\beta)}=\bigcup_{\sigma \in \mathcal{F}_{K}} D_{K, \sigma}^{(\beta)},
\end{aligned}
$$

(note that we have $\left.\left|D_{K, \sigma} \backslash D_{K, \sigma}^{(\beta)}\right|=\frac{1-\beta^{d}}{d} d_{K, \sigma}|\sigma|\right)$.

Remark 3.4: The above definition for the discrete gradient $\nabla_{\mathcal{D}} v$ can be seen as a regularisation of the gradient of $v$ in the distribution sense, by replacing the Dirac mass on the faces of the mesh by a function with integral equal to 1.

Remark 3.5: It is possible to consider $\beta_{K, \sigma}$ instead of a constant $\beta$, without changing the mathematical analysis done in this paper.

Remark 3.6 (Piecewise constant reconstruction): One can for example replace $\Pi_{\mathcal{D}}$ by $\widehat{\Pi}_{\mathcal{D}}$ such that, for all $K \in \mathcal{M}$, and a.e. $\boldsymbol{x} \in K, \widehat{\Pi}_{\mathcal{D}} v(\boldsymbol{x})=\frac{1}{|K|} \int_{K} \bar{\Pi}_{K} v(\boldsymbol{x}) \mathrm{d} \boldsymbol{x}$, which provides a piecewise constant reconstruction in the sense of Definition 2.6, choosing a basis $\left(\chi_{i}\right)_{i \in I}$ such that, for each $K \in \mathcal{M}$, there exists $i \in I$ with $v_{i}=\frac{1}{|K|} \int_{K} \bar{\Pi}_{K} v(\boldsymbol{x}) \mathrm{d} \boldsymbol{x}$ for all $v \in X_{\mathcal{D}, 0}$.

Remark 3.7 (Definition of the jump at the faces of the mesh): Note that, in Definition 3.2, the jump at the faces is divided by 2 at the interior faces. This allows to keep the same definition for $\nabla_{\mathcal{D}}$ on all $D_{K, \sigma}$ in the two cases, $\sigma \in \mathcal{F}_{\text {int }}$ and $\sigma \in \mathcal{F}_{\text {ext }}$.

\subsection{Mathematical properties of the DGGD method}

Let us first state and prove the following lemma, which provides a connection between the discrete gradient defined by (15) to a norm suited for the study of discontinuous Galerkin methods in the framework of elliptic problems.

Lemma 3.8: Let $\mathcal{D}$ be a DGGD in the sense of Definition 3.2. Then there exists $A>0$, depending only on $p, k$ and $d$, such that

$$
\forall v \in X_{\mathcal{D}, 0}, \frac{\beta^{\frac{d}{p}+(k-1)}}{A}\|v\|_{\mathrm{DG}, p} \leq\left\|\nabla_{\mathcal{D}} v\right\|_{L^{p}(\Omega)^{d}} \leq \frac{A}{1-\beta}\|v\|_{\mathrm{DG}, p}
$$

where

$$
\|v\|_{\mathrm{DG}, p}^{p}=\sum_{K \in \mathcal{M}}\left(\int_{K}\left|\nabla_{\bar{K}} v(\boldsymbol{x})\right|^{p} \mathrm{~d} \boldsymbol{x}+\sum_{\sigma \in \mathcal{F}_{K}} \frac{1}{d_{K, \sigma}^{p-1}} \int_{\sigma}\left|[v]_{K, \sigma}(\boldsymbol{y})\right|^{p} \mathrm{~d} \gamma(\boldsymbol{y})\right) .
$$


Remark 3.9 (DG norm): Note that Definition (21) for the DG norm is slightly different from [7, eqn. (5)] or [8, eqn. (5.1)], with the use of $d_{K, \sigma}$ instead that of $\operatorname{diam}(\sigma)$, and with notation (16) for the jump at the faces of the mesh, owing to the fact that we make the assumption that the grid cells are star-shaped with respect to a point (this assumption is not made in $[7,8]$ ). This allows the application of discrete functional analysis results without regularity hypotheses on the polytopal mesh.

Proof. Let $K \in \mathcal{M}$ and $\sigma \in \mathcal{F}_{K}$. Using $\boldsymbol{x}=\boldsymbol{x}_{K}+s\left(\boldsymbol{y}-\boldsymbol{x}_{K}\right)$, and using, for some $c>0$ to be chosen later, $|a+b|^{p} \leq\left(1+c^{p^{\prime}}\right)^{p-1}\left(|a|^{p}+\left|\frac{b}{c}\right|^{p}\right)$ with $\frac{1}{p}+\frac{1}{p^{\prime}}=1, a=\nabla_{\bar{K}} v(\boldsymbol{x})+\frac{[v]_{K, \sigma}(\boldsymbol{Y}(\boldsymbol{x}))}{d_{K, \sigma}} \psi(S(\boldsymbol{x})) \boldsymbol{n}_{K, \sigma}$ and $b=-\nabla_{\bar{K}} v(\boldsymbol{x})$, we have

$$
\begin{aligned}
\int_{K}\left|\nabla_{\mathcal{D}} v(\boldsymbol{x})\right|^{p} \mathrm{~d} \boldsymbol{x}= & \int_{K^{(\beta)}}\left|\nabla_{\bar{K}} v(\boldsymbol{x})\right|^{p} \mathrm{~d} \boldsymbol{x}+\sum_{\sigma \in \mathcal{F}_{K}} \int_{D_{K, \sigma} \backslash D_{K, \sigma}^{(\beta)}}\left|\nabla_{\bar{K}} v(\boldsymbol{x})+\frac{[v]_{K, \sigma}(\boldsymbol{Y}(\boldsymbol{x}))}{d_{K, \sigma}} \psi(S(\boldsymbol{x})) \boldsymbol{n}_{K, \sigma}\right|^{p} \mathrm{~d} \boldsymbol{x} \\
\geq & \int_{K^{(\beta)}}\left|\nabla_{\bar{K}} v(\boldsymbol{x})\right|^{p} \mathrm{~d} \boldsymbol{x}-\frac{1}{c^{p}} \int_{K \backslash K(\beta)}\left|\nabla_{\bar{K}} v(\boldsymbol{x})\right|^{p} \mathrm{~d} \boldsymbol{x} \\
& +\frac{1}{\left(1+c^{p^{\prime}}\right)^{p-1}} \sum_{\sigma \in \mathcal{F}_{K}} \int_{D_{K, \sigma} \backslash D_{K, \sigma}^{(\beta)}}\left|\frac{[v]_{K, \sigma}(\boldsymbol{Y}(\boldsymbol{x}))}{d_{K, \sigma}} \psi(S(\boldsymbol{x})) \boldsymbol{n}_{K, \sigma}\right|^{p} \mathrm{~d} \boldsymbol{x} .
\end{aligned}
$$

Writing

$$
\frac{1}{d} \sum_{i=1}^{d}\left|\partial_{i} \Pi_{\bar{K}} v\right|^{p} \leq\left|\nabla_{\bar{K}} v\right|^{p} \leq d^{p / 2} \sum_{i=1}^{d}\left|\partial_{i} \Pi_{\bar{K}} v\right|^{p}
$$

and applying Lemma A.3 to the polynomial $\partial_{i} \Pi_{\bar{K}} v \in \mathbb{P}_{k-1}\left(\mathbb{R}^{d}\right)$ for $i=1, \ldots, d$, we can write

$$
\int_{K^{(\beta)}}\left|\nabla_{\bar{K}} v(\boldsymbol{x})\right|^{p} \mathrm{~d} \boldsymbol{x} \geq \frac{\beta^{d+p(k-1)} C_{p, k-1}}{d k^{p-1}} \sum_{i=1}^{d} \int_{K}\left|\partial_{i} \Pi_{\bar{K}} v(\boldsymbol{x})\right|^{p} \mathrm{~d} \boldsymbol{x} \geq 2 \beta^{d+p(k-1)} C_{p, k}^{\prime} \int_{K}\left|\nabla_{\bar{K}} v(\boldsymbol{x})\right|^{p} \mathrm{~d} \boldsymbol{x},
$$

denoting by $C_{p, k}^{\prime}=\frac{C_{p, k-1}}{2 d^{1+p / 2} k^{p-1}}$. We then define $c$ by $\frac{1}{c^{p}}=\beta^{d+p(k-1)} C_{p, k}^{\prime}$. Remarking that

$$
\frac{1}{\left(1+c^{p^{\prime}}\right)^{p-1}}=\frac{\beta^{d+p(k-1)} C_{p, k}^{\prime}}{\left(\left(\beta^{d+p(k-1)} C_{p, k}^{\prime}\right)^{p^{\prime} / p}+1\right)^{p-1}} \geq \beta^{d+p(k-1)} \frac{C_{p, k}^{\prime}}{\left(\left(C_{p, k}^{\prime}\right)^{p^{\prime} / p}+1\right)^{p-1}},
$$

and using $\left|\boldsymbol{n}_{K, \sigma}\right|=1$, we get

$$
\begin{aligned}
\int_{K}\left|\nabla_{\mathcal{D}} v(\boldsymbol{x})\right|^{p} \mathrm{~d} \boldsymbol{x} \geq \beta^{d+p(k-1)}( & C_{p, k}^{\prime} \int_{K}\left|\nabla_{\bar{K}} v(\boldsymbol{x})\right|^{p} \mathrm{~d} \boldsymbol{x} \\
& \left.+\frac{C_{p, k}^{\prime}}{\left(\left(C_{p, k}^{\prime}\right)^{p^{\prime} / p}+1\right)^{p-1}} \sum_{\sigma \in \mathcal{F}_{K}} \int_{D_{K, \sigma} \backslash D_{K, \sigma}^{(\beta)}}\left|\frac{[v]_{K, \sigma}(\boldsymbol{Y}(\boldsymbol{x}))}{d_{K, \sigma}} \psi(S(\boldsymbol{x}))\right|^{p} \mathrm{~d} \boldsymbol{x}\right) .
\end{aligned}
$$

We then apply the change of variable $\boldsymbol{x} \in D_{K, \sigma} \mapsto(\boldsymbol{y}=\boldsymbol{Y}(\boldsymbol{x}), s=S(\boldsymbol{x})) \in \sigma \times(0,1)$. We then have $\boldsymbol{x}=\boldsymbol{x}_{K}+s\left(\boldsymbol{y}-\boldsymbol{x}_{K}\right)$ and therefore $\mathrm{d} \boldsymbol{x}=d_{K, \sigma} s^{d-1} \mathrm{~d} \gamma(\boldsymbol{y}) \mathrm{d} s$, leading to

$$
\begin{aligned}
\int_{D_{K, \sigma} \backslash D_{K, \sigma}^{(\beta)}}\left|\frac{[v]_{K, \sigma}(\boldsymbol{Y}(\boldsymbol{x}))}{d_{K, \sigma}} \psi(S(\boldsymbol{x}))\right|^{p} \mathrm{~d} \boldsymbol{x} & =d_{K, \sigma} \int_{\sigma} \frac{\left|[v]_{K, \sigma}(\boldsymbol{y})\right|^{p}}{d_{K, \sigma}^{p}} \mathrm{~d} \gamma(\boldsymbol{y}) \int_{\beta}^{1}|\psi(s)|^{p} s^{d-1} \mathrm{~d} s \\
& =\frac{1}{d_{K, \sigma}^{p-1}} \int_{\sigma}\left|[v]_{K, \sigma}(\boldsymbol{y})\right|^{p} \mathrm{~d} \gamma(\boldsymbol{y}) \int_{\beta}^{1}|\psi(s)|^{p} s^{d-1} \mathrm{~d} s .
\end{aligned}
$$

Using (17) and Hölder's inequality, we have

$$
1=\int_{\beta}^{1} \psi(s) s^{d-1} \mathrm{~d} s \leq\left(\int_{\beta}^{1}|\psi(s)|^{p} s^{d-1} \mathrm{~d} s\right)^{\frac{1}{p}}\left(\int_{\beta}^{1} s^{d-1} \mathrm{~d} s\right)^{\frac{1}{p^{\prime}}}=\left(\int_{\beta}^{1}|\psi(s)|^{p} s^{d-1} \mathrm{~d} s\right)^{\frac{1}{p}}\left(\frac{1-\beta^{d}}{d}\right)^{\frac{1}{p^{\prime}}}
$$


leading to

$$
\int_{\beta}^{1}|\psi(s)|^{p} s^{d-1} \mathrm{~d} s \geq\left(\frac{d}{1-\beta^{d}}\right)^{p-1} \geq d^{p-1} .
$$

This completes the proof of the left inequality in (20). Turning to the proof of the right inequality of (20), we have

$$
\begin{aligned}
\int_{D_{K, \sigma}}\left|\nabla_{\mathcal{D}} v(\boldsymbol{x})\right|^{p} \mathrm{~d} \boldsymbol{x} & \leq 2^{p-1} \int_{D_{K, \sigma}}\left|\nabla_{\bar{K}} v(\boldsymbol{x})\right|^{p} \mathrm{~d} \boldsymbol{x}+2^{p-1} \int_{D_{K, \sigma} \backslash D_{K, \sigma}^{(\beta)}}\left|\frac{[v]_{K, \sigma}(\boldsymbol{Y}(\boldsymbol{x}))}{d_{K, \sigma}} \psi(S(\boldsymbol{x}))\right|^{p} \mathrm{~d} \boldsymbol{x} \\
& =2^{p-1} \int_{D_{K, \sigma}}\left|\nabla_{\bar{K}} v(\boldsymbol{x})\right|^{p} \mathrm{~d} \boldsymbol{x}+\left(\frac{2}{d_{K, \sigma}}\right)^{p-1} \int_{\sigma}\left|[v]_{K, \sigma}(\boldsymbol{y})\right|^{p} \mathrm{~d} \gamma(\boldsymbol{y}) \int_{\beta}^{1}|\psi(s)|^{p} s^{d-1} \mathrm{~d} s
\end{aligned}
$$

which completes the proof of the right inequality in (20), since, using (57),

$$
\int_{\beta}^{1}|\psi(s)|^{p} s^{d-1} \mathrm{~d} s \leq \frac{\left(1-\beta^{d}\right) C^{p}}{d(1-\beta)^{p}} \leq \frac{C^{p}}{d(1-\beta)^{p}} .
$$

We can now state and prove that $\left\|\nabla_{\mathcal{D}} \cdot\right\|_{L^{p}(\Omega)^{d}}$ is a norm on $X_{\mathcal{D}, 0}$.

Lemma 3.10: Let $\mathcal{D}$ be a DGGD in the sense of Definition 3.2. Then $\left\|\nabla_{\mathcal{D}} \cdot\right\|_{L^{p}(\Omega) d}$ is a norm on $X_{\mathcal{D}, 0}$.

Proof. It suffices to check that, if $v \in X_{\mathcal{D}, 0}$ is such that $\left\|\nabla_{\mathcal{D}} v\right\|_{L^{p}(\Omega) d}=0$, then $v=0$. Indeed, owing to Lemma 3.8, we get that $\|v\|_{\mathrm{DG}, p}=0$. We can apply Lemma A.7, since in the case $d=1$ or $d=2$, it applies without restriction to $q=p$, and in the case $d \geq 3$, there holds $p<p^{\star}=p d /(d-p)$. Hence we deduce that $\left\|\Pi_{\mathcal{D}} v\right\|_{L^{p}(\Omega)}=0$, which concludes the proof.

We now prove the core properties of the DGGD, as described in Section 2, gathered in the following theorem.

Theorem 3.11 (Properties of DGGDs): Let $\left(\mathcal{D}_{m}\right)_{m \in \mathbb{N}}$ be a sequence of DGGDs in the sense of Definition 3.2, defined from underlying polytopal meshes $\left(\mathfrak{T}_{m}\right)_{m \in \mathbb{N}}$. Assume that $\left(\eta_{\mathfrak{T}_{m}}\right)_{m \in \mathbb{N}}$ is bounded (see $(11)$ ), and that $h_{\mathcal{M}_{m}} \rightarrow 0$ as $m \rightarrow \infty$.

Then the sequence $\left(\mathcal{D}_{m}\right)_{m \in \mathbb{N}}$ is coercive, GD-consistent, limit-conforming and compact in the sense of Definitions 2.2, 2.3, 2.4 and 2.5.

Proof. The limit-conformity, coercivity, GD-consistency and compactness are obtained by applying Lemmas 3.15, 3.12, 3.14, 3.13 .

Let us now prove each of the lemmas involved in the proof of the above theorem.

Lemma 3.12 (Coercivity): Let $\mathcal{D}$ be a DGGD in the sense of Definition 3.2. Let $C_{\mathcal{D}} \geq 0$ be defined by (2). Then there exists $C_{P}$ depending only on $|\Omega|, \beta, p, k$ and $d$ such that $C_{P} \geq C_{\mathcal{D}}$, which means that any sequence $(\mathcal{D})_{m \in \mathbb{N}}$ is coercive in the sense of Definition 2.2 .

Proof. We again apply Lemma A.7, since we noticed in the proof of Lemma 3.10 that it applies to the case $q=p$.

Lemma 3.13 (Compactness): Let $(\mathcal{D})_{m \in \mathbb{N}}$ be a sequence of DGGDs in the sense of Definition 3.2. Then, for all $\left(v_{m}\right)_{m \in \mathbb{N}}$ such that, for all $m \in \mathbb{N}, v_{m} \in X_{\mathcal{D}_{m}, 0}$ and such that the sequence $\left(\left\|\nabla_{\mathcal{D}_{m}} v_{m}\right\|_{L^{p}(\Omega)}\right)_{m \in \mathbb{N}}$ is bounded, the sequence $\left(\Pi_{\mathcal{D}_{m}} v_{m}\right)_{m \in \mathbb{N}}$ is relatively compact in $L^{p}(\Omega)$, which means that any sequence $\left(\mathcal{D}_{m}\right)_{m \in \mathbb{N}}$ is compact in the sense of Definition 2.5.

Proof. We first extend $\Pi_{\mathcal{D}_{m}} v_{m}$ by 0 in $\mathbb{R}^{d} \backslash \Omega$. We then have, for all $m \in \mathbb{N}$, applying Lemma A.6,

$$
\begin{array}{r}
\forall \xi \in \mathbb{R}^{d},\left\|\Pi_{\mathcal{D}_{m}} v_{m}(.+\xi)-\Pi_{\mathcal{D}_{m}} v_{m}\right\|_{L^{1}\left(\mathbb{R}^{d}\right)} \leq\left(\sum_{i=1}^{d}\left|\xi_{i}\right|\right)\left\|\Pi_{\mathcal{D}_{m}} v_{m}\right\|_{\mathrm{BV}} \\
\leq\left(\sum_{i=1}^{d}\left|\xi_{i}\right|\right) C d((d+1)|\Omega|)^{\frac{p-1}{p}}\left\|\Pi_{\mathcal{D}_{m}} v_{m}\right\|_{\mathrm{DG}, p} .
\end{array}
$$


This implies that the sequence $\left(\Pi_{\mathcal{D}_{m}} v_{m}\right)_{m \in \mathbb{N}}$ is relatively compact in $L^{1}\left(\mathbb{R}^{d}\right)$. Thanks to Lemma A.7, the sequence $\left(\Pi_{\mathcal{D}_{m}} v_{m}\right)_{m \in \mathbb{N}}$ is bounded in $L^{q}\left(\mathbb{R}^{d}\right)$ for some $q>p$. We thus deduce that the sequence $\left(\Pi_{\mathcal{D}_{m}} v_{m}\right)_{m \in \mathbb{N}}$ is relatively compact in $L^{p}\left(\mathbb{R}^{d}\right)$, which concludes the proof of the lemma.

Lemma 3.14 (Estimate on $S_{\mathcal{D}}$ for DGGD): Let $\mathcal{D}$ be a DGGD in the sense of Definition 3.2, with underlying polytopal mesh $\mathfrak{T}$. Take $\varrho \geq \eta_{\mathfrak{T}}$ (see $(11)$ ) and let $\ell \in\{1, \ldots, k\}$. Then there exists $C_{1}>0$, depending only on on $\Omega, \beta, p, k, \ell, d$ and $\varrho$, such that

$$
\forall \varphi \in W^{\ell+1, p}(\Omega) \cap W_{0}^{1, p}(\Omega), S_{\mathcal{D}}(\varphi) \leq C_{1} h_{\mathcal{M}}^{\ell}\|\varphi\|_{W^{\ell+1, p}(\Omega)},
$$

where $S_{\mathcal{D}}$ is defined by (3).

As a consequence, any sequence $(\mathcal{D})_{m \in \mathbb{N}}$ of DGGDs such that $h_{\mathfrak{T}_{m}}$ tends to 0 as $m \rightarrow \infty$ while $\eta_{\mathfrak{T}_{m}}$ remains bounded is GD-consistent in the sense of Definition 2.3.

Proof. In this proof, $C$ is a generic notation for various positive numbers depending only on $\Omega, \beta, p$, $k, \ell, d$ and $\varrho$. Let $\varphi \in W^{\ell+1, p}(\Omega) \cap W_{0}^{1, p}(\Omega)$ and, for $K \in \mathcal{M}$, denote by $\pi_{K}^{k}: L^{1}(K) \rightarrow \mathbb{P}_{k}(K)$ the $L^{2}(K)$-projection on polynomials over $K$ of degree at most $k$. By [6, Lemmata 3.4 and 3.6],

$$
\begin{aligned}
&\left\|\varphi-\pi_{K}^{k} \varphi\right\|_{L^{p}(K)} \leq C h_{K}^{\ell+1}\|\varphi\|_{W^{\ell+1, p}(K)}, \\
&\left\|\nabla \varphi-\nabla\left(\pi_{K}^{k} \varphi\right)\right\|_{L^{p}(K)^{d}} \leq C h_{K}^{\ell}\|\varphi\|_{W^{\ell+1, p}(K)}, \\
& \forall \sigma \in \mathcal{F}_{K},\left\|\varphi-\pi_{K}^{k} \varphi\right\|_{L^{p}(\sigma)} \leq C h_{K}^{\ell+1-\frac{1}{p}}\|\varphi\|_{W^{\ell+1, p}(K)} .
\end{aligned}
$$

The functions $\left(\pi_{K}^{k} \varphi\right)_{K \in \mathcal{M}}$ define an element of $W_{h}$. Since $\Pi_{\mathcal{D}}: X_{\mathcal{D}, 0} \rightarrow W_{h}$ is an isomorphism (see its definition (14)), there exists $v \in X_{\mathcal{D}, 0}$ such that $\left(\Pi_{\mathcal{D}} v\right)_{\mid K}=\Pi_{\bar{K}} v=\pi_{K}^{k} \varphi$ for all $K \in \mathcal{M}$. Then, raising (24) to the power $p$ and summing over $K \in \mathcal{M}$ yields

$$
\left\|\varphi-\Pi_{\mathcal{D}} v\right\|_{L^{p}(\Omega)} \leq C h_{\mathcal{M}}^{\ell+1}\|\varphi\|_{W^{\ell+1, p}(\Omega)} .
$$

Let us now analyse the jump terms in $\nabla_{\mathcal{D}} v$. Let $\sigma \in \mathcal{F}_{\text {int }}$ with $\mathcal{M}_{\sigma}=\{K, L\}$, and $\boldsymbol{y} \in \sigma$. Writing

$$
[v]_{K, \sigma}(\boldsymbol{y})=\frac{1}{2}\left(\Pi_{\bar{L}} v(\boldsymbol{y})-\varphi(\boldsymbol{y})+\varphi(\boldsymbol{y})-\Pi_{\bar{K}} v(\boldsymbol{y})\right)=\frac{1}{2}\left(\pi_{L}^{k} \varphi(\boldsymbol{y})-\varphi(\boldsymbol{y})\right)+\frac{1}{2}\left(\varphi(\boldsymbol{y})-\pi_{K}^{k} \varphi(\boldsymbol{y})\right),
$$

and using (26) in cells $K$ and $L$ yields

$$
\begin{aligned}
\left\|[v]_{K, \sigma}\right\|_{L^{p}(\sigma)} & \leq C h_{L}^{\ell+1-\frac{1}{p}}\|\varphi\|_{W^{\ell+1, p}(L)}+C h_{K}^{\ell+1-\frac{1}{p}}\|\varphi\|_{W^{\ell+1, p}(K)} \\
& \leq C h_{\mathcal{M}}^{\ell}\left(h_{L}^{1-\frac{1}{p}}\|\varphi\|_{W^{\ell+1, p}(L)}+h_{K}^{1-\frac{1}{p}}\|\varphi\|_{W^{\ell+1, p}(K)}\right) .
\end{aligned}
$$

By definition of $\varrho, d_{K, \sigma} \geq \varrho^{-1} h_{K}$ and $d_{K, \sigma} \geq \varrho^{-1} d_{L, \sigma} \geq \varrho^{-2} h_{L}$, so $d_{K, \sigma}^{\frac{1}{p}-1} \leq C h_{K}^{\frac{1}{p}-1}$ and $d_{K, \sigma}^{\frac{1}{p}-1} \leq C h_{L}^{\frac{1}{p}-1}$. Hence,

$$
d_{K, \sigma}^{\frac{1}{p}-1}\left\|[v]_{K, \sigma}\right\|_{L^{p}(\sigma)} \leq C h_{\mathcal{M}}^{\ell}\left(\|\varphi\|_{W^{\ell+1, p}(L)}+\|\varphi\|_{W^{\ell+1, p}(K)}\right) .
$$

Using change of variable $\boldsymbol{x} \mapsto(\boldsymbol{y}=\boldsymbol{Y}(\boldsymbol{x}), s=S(\boldsymbol{x}))$, we infer

$$
\begin{aligned}
& \int_{D_{K, \sigma}}\left|\nabla_{\mathcal{D}} v(\boldsymbol{x})-\nabla_{\bar{K}} v(\boldsymbol{x})\right|^{p} \mathrm{~d} \boldsymbol{x}=\int_{D_{K, \sigma}}\left|\psi(S(\boldsymbol{x})) \frac{[v]_{K, \sigma}(\boldsymbol{Y}(\boldsymbol{x}))}{d_{K, \sigma}}\right|^{p} \mathrm{~d} \boldsymbol{x} \\
& =\int_{\beta}^{1}|\psi(s)|^{p} s^{d-1} \mathrm{~d} s \int_{\sigma} d_{K, \sigma}^{1-p}\left|[v]_{K, \sigma}(\boldsymbol{y})\right|^{p} \mathrm{~d} \gamma(\boldsymbol{y}) \leq C h_{\mathcal{M}}^{p \ell}\left(\|\varphi\|_{W^{\ell+1, p}(L)}^{p}+\|\varphi\|_{W^{\ell+1, p(K)}}^{p}\right) .
\end{aligned}
$$

Since $\varphi=0$ on $\partial \Omega$, performing the same steps as above shows that (28) also holds if $\sigma \in \mathcal{F}_{K} \cap \mathcal{F}_{\text {ext }}$, by removing the term involving $L$. By summing (28) over $\sigma \in \mathcal{F}_{K}$ and $K \in \mathcal{M}$, using the definition $\nabla_{\bar{K}} v=\nabla\left(\Pi_{\bar{K}} v\right)=\nabla\left(\pi_{K}^{k} \varphi\right),(25)$ and the triangle inequality, we infer

$$
\left\|\nabla_{\mathcal{D}} v-\nabla \varphi\right\|_{L^{p}(\Omega)^{d}} \leq C h_{\mathcal{M}}^{\ell}\|\varphi\|_{W^{\ell+1, p}(\Omega)} .
$$


Combined with (27), this completes the proof of (23).

We then show that any sequence $(\mathcal{D})_{m \in \mathbb{N}}$ of DGGDs such that $h_{\mathfrak{T}_{m}}$ tends to 0 as $m \rightarrow \infty$ while $\eta_{\mathfrak{T}_{m}}$ remain bounded is GD-consistent in the sense of Definition 2.3, by applying [12, Lemma 2.4] since $W^{\ell+1, p}(\Omega) \cap$ $W_{0}^{1, p}(\Omega)$ is dense in $W_{0}^{1, p}(\Omega)$.

Lemma 3.15 (Estimate on $W_{\mathcal{D}}(\boldsymbol{\varphi})$ for DGGD): Let $\mathcal{D}$ be a DGGD in the sense of Definition 3.2 , with underlying polytopal mesh $\mathfrak{T}$. Take $\varrho \geq \eta_{\mathfrak{T}}$ (see $(11)$ ) and let $\ell \in\{1, \ldots, k\}$. Then there exists $C_{2}$, depending only on $\Omega, \beta, p, k, \ell, d$ and $\varrho$, such that

$$
\forall \varphi \in W^{\ell, p^{\prime}}(\Omega)^{d}, W_{\mathcal{D}}(\varphi) \leq C_{2} h_{\mathcal{M}}^{\ell}\|\varphi\|_{W^{\ell, p^{\prime}}(\Omega)^{d}},
$$

where $W_{\mathcal{D}}$ is defined by $(6)$.

As a consequence, any sequence $(\mathcal{D})_{m \in \mathbb{N}}$ of DGGDs in the sense of Definition 3.2 such that $h_{\mathfrak{T}_{m}}$ tends to 0 as $m \rightarrow \infty$ is limit-conforming in the sense of Definition 2.4.

Proof. In this proof, $C$ denotes various constants having the same dependencies as $C_{2}$ in the lemma. Let $\varphi \in W^{\ell, p^{\prime}}(\Omega)^{d}$. Using the definition of $\nabla_{\mathcal{D}} v$ and $\Pi_{\mathcal{D}} v$ yields

$$
\begin{aligned}
& \int_{\Omega}\left(\nabla_{\mathcal{D}} v(\boldsymbol{x}) \cdot \boldsymbol{\varphi}(\boldsymbol{x})+\Pi_{\mathcal{D}} v(\boldsymbol{x}) \operatorname{div} \boldsymbol{\varphi}(\boldsymbol{x})\right) \mathrm{d} \boldsymbol{x}=T_{1}+T_{2}, \\
\text { with } & T_{1}=\sum_{K \in \mathcal{M}} \int_{K}\left(\nabla\left(\Pi_{\bar{K}} v\right)(\boldsymbol{x}) \cdot \boldsymbol{\varphi}(\boldsymbol{x})+\Pi_{\bar{K}} v(\boldsymbol{x}) \operatorname{div} \boldsymbol{\varphi}(\boldsymbol{x})\right) \mathrm{d} \boldsymbol{x}, \\
\text { and } \quad & T_{2}=\sum_{K \in \mathcal{M}} \sum_{\sigma \in \mathcal{F}_{K}} \int_{D_{K, \sigma}} \psi(S(\boldsymbol{x})) \frac{[v]_{K, \sigma}(\boldsymbol{Y}(\boldsymbol{x}))}{d_{K, \sigma}} \boldsymbol{n}_{K, \sigma} \cdot \boldsymbol{\varphi}(\boldsymbol{x}) \mathrm{d} \boldsymbol{x} .
\end{aligned}
$$

Stokes formula in each cell $K$ yields

$$
T_{1}=\sum_{K \in \mathcal{M}} \sum_{\sigma \in \mathcal{F}_{K}} \int_{\sigma} \Pi_{\bar{K}} v(\boldsymbol{y}) \boldsymbol{\varphi}(\boldsymbol{y}) \cdot \boldsymbol{n}_{K, \sigma} \mathrm{d} \gamma(\boldsymbol{y}) .
$$

Let $\pi_{K}^{k-1}: L^{p}(K)^{d} \rightarrow \mathbb{P}_{k-1}(K)^{d}$ be the component-wise $L^{2}(K)$-projection over polynomial vectors on $K$ of degree at most $k-1$, and write

$$
\begin{array}{rrr} 
& T_{2}=T_{2,1}+T_{2,2}, \\
\text { with } & T_{2,1}=\sum_{K \in \mathcal{M}} \sum_{\sigma \in \mathcal{F}_{K}} \int_{D_{K, \sigma}} \psi(S(\boldsymbol{x})) \frac{[v]_{K, \sigma}(\boldsymbol{Y}(\boldsymbol{x}))}{d_{K, \sigma}} \boldsymbol{n}_{K, \sigma} \cdot\left(\boldsymbol{\varphi}(\boldsymbol{x})-\pi_{K}^{k-1} \boldsymbol{\varphi}(\boldsymbol{x})\right) \mathrm{d} \boldsymbol{x}, \\
\text { and } & T_{2,2}=\sum_{K \in \mathcal{M}} \sum_{\sigma \in \mathcal{F}_{K}} \int_{D_{K, \sigma}} \psi(S(\boldsymbol{x})) \frac{[v]_{K, \sigma}(\boldsymbol{Y}(\boldsymbol{x}))}{d_{K, \sigma}} \boldsymbol{n}_{K, \sigma} \cdot \pi_{K}^{k-1} \boldsymbol{\varphi}(\boldsymbol{x}) \mathrm{d} \boldsymbol{x} .
\end{array}
$$

Similarly to (24) we have $\left\|\varphi-\pi_{K}^{k-1} \varphi\right\|_{L^{p^{\prime}(K)^{d}}} \leq C h_{K}^{\ell}\|\varphi\|_{W^{\ell, p^{\prime}(K)^{d}}}$. Hence, owing to Hölder's inequalities and the change of variable $\boldsymbol{x} \mapsto(\boldsymbol{y}=\boldsymbol{Y}(\boldsymbol{x}), s=S(\boldsymbol{x}))$ and Lemma 3.8 , we get

$$
\begin{aligned}
\left|T_{2,1}\right| & \leq C h_{\mathcal{M}}^{\ell}\|\varphi\|_{W^{\ell, p^{\prime}}(\Omega)^{d}}\left(\sum_{K \in \mathcal{M}} \sum_{\sigma \in \mathcal{F}_{K}} \int_{D_{K, \sigma}}|\psi(s)|^{p} s^{d-1} \mathrm{~d} s \int_{\sigma} d_{K, \sigma}^{1-p}\left|[v]_{K, \sigma}(\boldsymbol{y})\right|^{p} \mathrm{~d} \gamma(\boldsymbol{y})\right)^{1 / p} \\
& \leq C h_{\mathcal{M}}^{\ell}\|\boldsymbol{\varphi}\|_{W^{\ell, p^{\prime}}(\Omega)^{d}}\|v\|_{\mathrm{DG}, p} \leq C A \beta^{-\left(\frac{d}{p}+(k-1)\right)} h_{\mathcal{M}}^{\ell}\|\boldsymbol{\varphi}\|_{W^{\ell, p^{\prime}(\Omega)^{d}}}\left\|\nabla_{\mathcal{D}} v\right\|_{L^{p}(\Omega)^{d}}
\end{aligned}
$$

We now turn to $T_{2,2}$. Since $\pi_{K}^{k-1} \varphi$ is a polynomial of degree $k-1$ or less, using the change of variable $\boldsymbol{x} \mapsto(\boldsymbol{y}=\boldsymbol{Y}(\boldsymbol{x}), s=S(\boldsymbol{x}))$ and a Taylor expansion in $s$ about $s=1$, we have

$$
\pi_{K}^{k-1} \boldsymbol{\varphi}(\boldsymbol{x}) \cdot \boldsymbol{n}_{K, \sigma}=\pi_{K}^{k-1} \boldsymbol{\varphi}(\boldsymbol{y}) \cdot \boldsymbol{n}_{K, \sigma}+\sum_{m=1}^{k-1} p_{m}(\boldsymbol{y})(1-s)^{m}
$$


where $p_{m}$ is a polynomial in $\boldsymbol{y}$. Hence, reporting this expression into that of $T_{2,2}$, the second term of the right hand side vanishes owing to (18), and the first term is equal, owing to (17), to

$$
T_{2,2}=\sum_{K \in \mathcal{M}} \sum_{\sigma \in \mathcal{F}_{K}} \int_{\sigma}[v]_{K, \sigma}(\boldsymbol{y}) \pi_{K}^{k-1} \boldsymbol{\varphi}(\boldsymbol{y}) \cdot \boldsymbol{n}_{K, \sigma} \mathrm{d} \boldsymbol{y} .
$$

Therefore, with (31),

$$
T_{1}+T_{2,2}=\sum_{K \in \mathcal{M}} \sum_{\sigma \in \mathcal{F}_{K}} \int_{\sigma}\left(\Pi_{\bar{K}} v(\boldsymbol{y}) \boldsymbol{\varphi}(\boldsymbol{y})+[v]_{K, \sigma}(\boldsymbol{y}) \pi_{K}^{k-1} \boldsymbol{\varphi}(\boldsymbol{y})\right) \cdot \boldsymbol{n}_{K, \sigma} \mathrm{d} \gamma(\boldsymbol{y})=T_{3}+T_{4},
$$

with

$$
\begin{aligned}
& T_{3}=\sum_{K \in \mathcal{M}} \sum_{\sigma \in \mathcal{F}_{K}} \int_{\sigma}\left(\Pi_{\bar{K}} v(\boldsymbol{y})+[v]_{K, \sigma}(\boldsymbol{y})\right) \boldsymbol{\varphi}(\boldsymbol{y}) \cdot \boldsymbol{n}_{K, \sigma} \mathrm{d} \gamma(\boldsymbol{y}), \\
& T_{4}=\sum_{K \in \mathcal{M}} \sum_{\sigma \in \mathcal{F}_{K}} \int_{\sigma}[v]_{K, \sigma}(\boldsymbol{y})\left(\pi_{K}^{k-1} \boldsymbol{\varphi}(\boldsymbol{y})-\boldsymbol{\varphi}(\boldsymbol{y})\right) \cdot \boldsymbol{n}_{K, \sigma} \mathrm{d} \gamma(\boldsymbol{y}) .
\end{aligned}
$$

If $\sigma \in \mathcal{F}_{\text {int }}$ with $\mathcal{M}_{\sigma}=\{K, L\}, \Pi_{\bar{K}} v(\boldsymbol{y})+[v]_{K, \sigma}(\boldsymbol{y})=\frac{1}{2}\left(\Pi_{\bar{K}} v(\boldsymbol{y})+\Pi_{\bar{L}} v(\boldsymbol{y})\right)$ and thus, since $\boldsymbol{n}_{K, \sigma}+\boldsymbol{n}_{L, \sigma}=0$,

$$
\int_{\sigma}\left(\Pi_{\bar{K}} v(\boldsymbol{y})+[v]_{K, \sigma}(\boldsymbol{y})\right) \boldsymbol{\varphi}(\boldsymbol{y}) \cdot \boldsymbol{n}_{K, \sigma} \mathrm{d} \gamma(\boldsymbol{y})+\int_{\sigma}\left(\Pi_{\bar{L}} v(\boldsymbol{y})+[v]_{L, \sigma}(\boldsymbol{y})\right) \boldsymbol{\varphi}(\boldsymbol{y}) \cdot \boldsymbol{n}_{L, \sigma} \mathrm{d} \gamma(\boldsymbol{y})=0 .
$$

If $\sigma \in \mathcal{F}_{\text {ext }}$ with $\mathcal{M}_{\sigma}=\{K\}$, then $\Pi_{\bar{K}} v(\boldsymbol{y})+[v]_{K, \sigma}(\boldsymbol{y})=0$. These arguments show that $T_{3}=0$, and thus that $T_{1}+T_{2,2}=T_{4}$. Similarly to $(26)$, we have $\left\|\boldsymbol{\varphi}-\pi_{K}^{k-1} \boldsymbol{\varphi}\right\|_{L^{p^{\prime}}(\sigma)^{d}} \leq C h_{K}^{\ell-1 / p^{\prime}}\|\boldsymbol{\varphi}\|_{W^{\ell, p^{\prime}}(K)^{d}} \leq$ $C h_{\mathcal{M}}^{\ell} d_{K, \sigma}^{-1 / p^{\prime}}\|\varphi\|_{W^{\ell, p^{\prime}(K)^{d}}}$ (use $d_{K, \sigma} \leq h_{K}$ ). The Hölder's inequalities thus give

$$
\begin{aligned}
\left|T_{4}\right| & \leq C\left(\sum_{K \in \mathcal{M}} \sum_{\sigma \in \mathcal{F}_{K}} \int_{\sigma} d_{K, \sigma}^{1-p}\left|[v]_{K, \sigma}(\boldsymbol{y})\right|^{p} \mathrm{~d} \gamma(\boldsymbol{y})\right)^{1 / p} h_{\mathcal{M}}^{\ell}\|\boldsymbol{\varphi}\|_{W^{\ell, p^{\prime}(\Omega)^{d}}} \\
& \leq C\|v\|_{\mathrm{DG}, p} h_{\mathcal{M}}^{\ell}\|\boldsymbol{\varphi}\|_{W^{\ell, p^{\prime}(\Omega)^{d}}} \\
& \leq C A \beta^{-\left(\frac{d}{p}+(k-1)\right)} h_{\mathcal{M}}^{\ell}\left\|\nabla_{\mathcal{D}} v\right\|_{L^{p}(\Omega)^{d}}\|\boldsymbol{\varphi}\|_{W^{\ell, p^{\prime}(\Omega)^{d}}} .
\end{aligned}
$$

Combined with (33) and plugged alongside (32) into (30), this concludes the proof of (29).

Then, considering a sequence $(\mathcal{D})_{m \in \mathbb{N}}$ of DGGDs in the sense of Definition 3.2 such that $h_{\mathfrak{T}_{m}}$ tends to 0 as $m \rightarrow \infty$, we get that it is limit-conforming in the sense of Definition 2.4 by density and applying [12, Lemma 2.4] (this is possible owing to Lemma 3.12 which states that $(\mathcal{D})_{m \in \mathbb{N}}$ is coercive).

Remark 3.16: Note that the application of Lemmas 3.14 and 3.15 to the error estimate [12, Lemma 2.2, eqns. (6) and (7)] provides an error in $h_{\mathcal{M}}$ in the case of the linear elliptic problem (52) under Hypotheses (53) in one, two or three space dimensions, when the exact solution belongs to $H^{2}(\Omega)$.

\section{Average discontinuous Galerkin gradient discretisation (ADGGD)}

This section presents a second gradient discretisation associated with discontinuous Galerkin methods. In this GD, called the Average Discontinuous Galerkin Gradient Discretisation (ADGGD), the discrete gradients are defined from average jumps instead of pointwise jumps. This idea can be found in [13] in the case of the elasticity operator (see Introduction), and in [3] with a non-symmetric scheme.

Let $X_{\mathcal{D}, 0}, \Pi_{\mathcal{D}}, \psi$ and $S$ be defined as in Definition 3.2 in Section 3. We substitute to (15)-(16) the following definition: for $v \in X_{\mathcal{D}, 0}, K \in \mathcal{M}, \sigma \in \mathcal{F}_{K}$ and a.e. $\boldsymbol{x} \in D_{K, \sigma}$, set

$$
\nabla_{\mathcal{D}} v(\boldsymbol{x})=\nabla_{\bar{K}} v(\boldsymbol{x})+\psi(S(\boldsymbol{x})) \frac{[v]_{K, \sigma}^{a}}{d_{K, \sigma}} \boldsymbol{n}_{K, \sigma},
$$


where the average jump $[v]_{K, \sigma}^{a}$, which replaces the pointwise jump in (15), is defined by

$$
\begin{aligned}
& \text { if } \sigma \in \mathcal{F}_{\text {int }} \text { and } \mathcal{M}_{\sigma}=\{K, L\},[v]_{K, \sigma}^{a}=\frac{1}{2}\left(v_{L, \sigma}^{a}-v_{K, \sigma}^{a}\right), \\
& \text { if } \sigma \in \mathcal{F}_{\text {ext }} \text { and } \mathcal{M}_{\sigma}=\{K\},[v]_{K, \sigma}^{a}=0-v_{K, \sigma}^{a},
\end{aligned}
$$
defining

$$
\forall K \in \mathcal{M}, \forall \sigma \in \mathcal{F}_{K}, v_{K, \sigma}^{a}=\frac{1}{|\sigma|} \int_{\sigma} \Pi_{\bar{K}} v(\boldsymbol{y}) \mathrm{d} \gamma(\boldsymbol{y}) .
$$

We remark that $\nabla_{\mathcal{D}} v$, as defined by (35)-(36) is piecewise constant in the case $k=1$. It is identical to that defined by (15)-(16) in the 1D case, letting $k=1$, but it leads to much simpler computations in the $2 \mathrm{D}$ or $3 \mathrm{D}$ cases (see Remark 5.2 in Section 5).

In order to prove that the GD such defined satisfies the properties listed in Section 2, we assume in the next lemmas that all the faces of the mesh are convex, which can easily be satisfied in the case of polytopal meshes: the faces being polytopes as well, they can be split into convex sub-faces if needed, without increasing the cost of the method (it does not enlarge the stencil). Let us first show the following result of comparison between pointwise and average values at the faces of the mesh.

Lemma 4.1: Let $\mathcal{D}$ be a DGGD in the sense of Definition 3.2, with underlying polytopal mesh $\mathfrak{T}$ such that all the faces $\sigma \in \mathcal{F}$ are convex. Let $\varrho \geq \eta_{\mathfrak{T}}$ (see (11)). Then there exists $C_{3}>0$, depending only on $p, k$, $\varrho$ and $d$, such that

$$
\forall v \in X_{\mathcal{D}, 0}, \forall K \in \mathcal{M}, \forall \sigma \in \mathcal{F}_{K}, \frac{1}{d_{K, \sigma}^{p-1}} \int_{\sigma}\left|\Pi_{\bar{K}} v(\boldsymbol{y})-v_{K, \sigma}^{a}\right|^{p} \mathrm{~d} \gamma(\boldsymbol{y}) \leq C_{3} \int_{D_{K, \sigma}}\left|\nabla_{\bar{K}} v(\boldsymbol{x})\right|^{p} \mathrm{~d} \boldsymbol{x} .
$$

Proof. Let us prove (37). Applying Jensen's inequality and to Fubini's theorem, we get

$$
\begin{aligned}
\int_{\sigma}\left|\Pi_{\bar{K}} v(\boldsymbol{y})-v_{K, \sigma}^{a}\right|^{p} \mathrm{~d} \gamma(\boldsymbol{y})=\int_{\sigma}\left|\frac{1}{|\sigma|} \int_{\sigma}\left(\Pi_{\bar{K}} v(\boldsymbol{y})-\Pi_{\bar{K}} v(\boldsymbol{x})\right) \mathrm{d} \gamma(\boldsymbol{x})\right|^{p} \mathrm{~d} \gamma(\boldsymbol{y}) & \\
& \leq \int_{\sigma} \frac{1}{|\sigma|} \int_{\sigma}\left|\Pi_{\bar{K}} v(\boldsymbol{y})-\Pi_{\bar{K}} v(\boldsymbol{x})\right|^{p} \mathrm{~d} \gamma(\boldsymbol{x}) \mathrm{d} \gamma(\boldsymbol{y})
\end{aligned}
$$

Thanks again to Jensen's inequality, we have

$$
\begin{array}{r}
\int_{\sigma} \frac{1}{|\sigma|} \int_{\sigma}\left|\Pi_{\bar{K}} v(\boldsymbol{y})-\Pi_{\bar{K}} v(\boldsymbol{x})\right|^{p} \mathrm{~d} \gamma(\boldsymbol{x}) \mathrm{d} \gamma(\boldsymbol{y})=\frac{1}{|\sigma|} \int_{\sigma} \int_{\sigma}\left|\int_{0}^{1} \nabla_{\bar{K}} v(\boldsymbol{x}+s(\boldsymbol{y}-\boldsymbol{x})) \cdot(\boldsymbol{y}-\boldsymbol{x}) \mathrm{d} s\right|^{p} \mathrm{~d} \gamma(\boldsymbol{x}) \mathrm{d} \gamma(\boldsymbol{y}) \\
\leq \frac{\operatorname{diam}(\sigma)^{p}}{|\sigma|} \int_{\sigma} \int_{\sigma} \int_{0}^{1}\left|\nabla_{\bar{K}} v(\boldsymbol{x}+s(\boldsymbol{y}-\boldsymbol{x}))\right|^{p} \mathrm{~d} s \mathrm{~d} \gamma(\boldsymbol{x}) \mathrm{d} \gamma(\boldsymbol{y}) \\
=\frac{\operatorname{diam}(\sigma)^{p}}{|\sigma|}\left(\int_{0}^{1 / 2} T_{1}(s) \mathrm{d} s+\int_{1 / 2}^{1} T_{1}(s) \mathrm{d} s\right)
\end{array}
$$

with

$$
T_{1}(s)=\int_{\sigma} \int_{\sigma}\left|\nabla_{\bar{K}} v((1-s) \boldsymbol{x}+s \boldsymbol{y})\right|^{p} \mathrm{~d} \gamma(\boldsymbol{x}) \mathrm{d} \gamma(\boldsymbol{y}) .
$$

We remark that, thanks the change of variable $s$ eqrefeqintintadgainLemma4.1to $(1-s)$ and exchanging $\boldsymbol{x}$ and $\boldsymbol{y}$, we can write

$$
\int_{1 / 2}^{1} T_{1}(s) \mathrm{d} s=\int_{0}^{1 / 2} T_{1}(s) \mathrm{d} s
$$

For a given $s \in\left(0, \frac{1}{2}\right)$, we make the change of variable $\boldsymbol{x} \rightarrow \boldsymbol{z}=(1-s) \boldsymbol{x}+s \boldsymbol{y} \in \sigma$ (recall that we assume that $\sigma$ is convex), and we denote by

$$
\sigma_{\boldsymbol{y}}=\{\boldsymbol{z} \in \sigma, \text { such that }(\boldsymbol{z}-s \boldsymbol{y}) /(1-s) \in \sigma\}
$$


We then have

$$
\begin{aligned}
& T_{1}(s)=\frac{1}{(1-s)^{d-1}} \int_{\sigma} \int_{\sigma_{\boldsymbol{y}}}\left|\nabla_{\bar{K}} v(\boldsymbol{z})\right|^{p} \mathrm{~d} \gamma(\boldsymbol{z}) \mathrm{d} \gamma(\boldsymbol{y}) \\
& \quad \leq 2^{d-1} \int_{\sigma} \int_{\sigma}\left|\nabla_{\bar{K}} v(\boldsymbol{z})\right|^{p} \mathrm{~d} \gamma(\boldsymbol{z}) \mathrm{d} \gamma(\boldsymbol{y})=|\sigma| 2^{d-1} \int_{\sigma}\left|\nabla_{\bar{K}} v(\boldsymbol{z})\right|^{p} \mathrm{~d} \gamma(\boldsymbol{z})
\end{aligned}
$$

Gathering the above results leads to

$$
\int_{\sigma}\left|\Pi_{\bar{K}} v(\boldsymbol{y})-v_{K, \sigma}^{a}\right|^{p} \mathrm{~d} \gamma(\boldsymbol{y}) \leq \operatorname{diam}(\sigma)^{p} 2^{d} \int_{\sigma}\left|\nabla_{\bar{K}} v(\boldsymbol{z})\right|^{p} \mathrm{~d} \gamma(\boldsymbol{z}) .
$$

For any $i=1, \ldots, d$, we then apply Lemma A.4 to $\partial_{i} \Pi_{\bar{K}} v$, which is a polynomial with degree lower or equal to $k-1$ with respect to $\boldsymbol{x} \in \mathbb{R}^{d}$. From (59), we then get

$$
d_{K, \sigma}\left\|\partial_{i} \Pi_{\bar{K}} v\right\|_{L^{p}(\sigma)}^{p} \leq \frac{k^{p-1}}{C_{p, k-1}}\left\|\partial_{i} \Pi_{\bar{K}} v\right\|_{L^{p}\left(D_{K, \sigma}\right)}^{p} \leq \frac{k^{p-1}}{C_{p, k-1}}\left\|\left|\nabla_{\bar{K}} v\right|\right\|_{L^{p}\left(D_{K, \sigma}\right)}^{p} .
$$

Since $\operatorname{diam}(\sigma) \leq h_{K} \leq d_{K, \sigma} \varrho$ by $(11)$, the two previous inequalities and the inequality

$$
\left\|\left|\nabla_{\bar{K}} v\right|\right\|_{L^{p}(\sigma)} \leq \sum_{i=1}^{d}\left\|\partial_{i} \Pi_{\bar{K}} v\right\|_{L^{p}(\sigma)}
$$

lead to (37).

Let us now show the following result of equivalence of norms, which enables the application of the Discrete Functional Analysis developed in the appendix.

Lemma 4.2: Let $\mathcal{D}$ be a DGGD in the sense of Definition 3.2, with underlying polytopal mesh $\mathfrak{T}$ such that all the faces $\sigma \in \mathcal{F}$ are convex. Let $\varrho \geq \eta_{\mathfrak{T}}$ (see (11)). For any $v \in X_{\mathcal{D}, 0}$, let us define $\|v\|_{\mathrm{ADG}, p}$ by

$$
\|v\|_{\mathrm{ADG}, p}^{p}=\sum_{K \in \mathcal{M}}\left(\int_{K}\left|\nabla_{\bar{K}} v(\boldsymbol{x})\right|^{p} \mathrm{~d} \boldsymbol{x}+\sum_{\sigma \in \mathcal{F}_{K}} \frac{|\sigma|}{d_{K, \sigma}^{p-1}}\left|[v]_{K, \sigma}^{a}\right|^{p}\right),
$$

(see (36) for the definition of $[v]_{K, \sigma}^{a}$ ). Then there exists $B>0$, depending only on $p, k, \varrho$ and $d$, such that

$$
\forall v \in X_{\mathcal{D}, 0},\|v\|_{\mathrm{ADG}, p} \leq\|v\|_{\mathrm{DG}, p} \leq B\|v\|_{\mathrm{ADG}, p},
$$

where $\|v\|_{\mathrm{DG}, p}$ is defined by (21).

Proof. We first remark on one hand that Jensen's inequality implies, for any $K \in \mathcal{M}$ and $\sigma \in \mathcal{F}_{K}$,

$$
|\sigma|\left|[v]_{K, \sigma}^{a}\right|^{p} \leq \int_{\sigma}\left|[v]_{K, \sigma}(\boldsymbol{y})\right|^{p} \mathrm{~d} \gamma(\boldsymbol{y}),
$$

which leads to the left inequality in (39), and on the other hand that, if $\sigma \in \mathcal{F}_{\text {int }}$ and $\mathcal{M}_{\sigma}=\{K, L\}$, we have

$$
\begin{aligned}
& \int_{\sigma}\left|[v]_{K, \sigma}(\boldsymbol{y})\right|^{p} \mathrm{~d} \gamma(\boldsymbol{y}) \\
& \quad \leq 3^{p-1}\left(\int_{\sigma}\left|\frac{1}{2}\left(\Pi_{\bar{K}} v(\boldsymbol{y})-v_{K, \sigma}^{a}\right)\right|^{p} \mathrm{~d} \gamma(\boldsymbol{y})+|\sigma|\left|[v]_{K, \sigma}^{a}\right|^{p}+\left.\int_{\sigma}\left|\frac{1}{2}\left(\Pi_{\bar{L}} v(\boldsymbol{y})-v_{L, \sigma}^{a}\right)\right|^{p} \mathrm{~d} \gamma(\boldsymbol{y})\right|^{p}\right)
\end{aligned}
$$

and if $\sigma \in \mathcal{F}_{\text {ext }}$ and $\mathcal{M}_{\sigma}=\{K\}$, there holds

$$
\int_{\sigma}\left|[v]_{K, \sigma}(\boldsymbol{y})\right|^{p} \mathrm{~d} \gamma(\boldsymbol{y}) \leq 2^{p-1}\left(\int_{\sigma}\left|\Pi_{\bar{K}} v(\boldsymbol{y})-v_{K, \sigma}^{a}\right|^{p} \mathrm{~d} \gamma(\boldsymbol{y})+|\sigma|\left|[v]_{K, \sigma}^{a}\right|^{p}\right),
$$

We then deduce the right inequality in (39), using (37) in Lemma 4.1.

We can now state and prove that $\left\|\nabla_{\mathcal{D}} \cdot\right\|_{L^{p}(\Omega)^{d}}$ is a norm on $X_{\mathcal{D}, 0}$. 
Lemma 4.3: Let $\mathcal{D}$ be a ADGGD in the sense of Definition 3.2, where (15)-(16) is replaced by (35)-(36), with underlying polytopal mesh $\mathfrak{T}$ such that all the faces $\sigma \in \mathcal{F}$ are convex. Then $\left\|\nabla_{\mathcal{D}} \cdot\right\|_{L^{p}(\Omega)^{d}}$ is a norm on $X_{\mathcal{D}, 0}$ equivalent to $\|\cdot\|_{\mathrm{DG}, p}$.

Proof. We first remark that, following the proof of Lemma 3.8 with replacing the pointwise jump by the average jump, we obtain the equivalence between $\left\|\nabla_{\mathcal{D}} \cdot\right\|_{L^{p}(\Omega) d}$ and $\|\cdot\|_{\mathrm{ADG}, p}$. The conclusion follows from Lemma 4.2.

We now state the core properties of the ADGGD, as described in Section 2, gathered in the following theorem.

Theorem 4.4 (Properties of ADGGDs): Let $\left(\mathcal{D}_{m}\right)_{m \in \mathbb{N}}$ be a sequence of ADGGDs in the sense of Definition 3.2 , where (15)-(16) is replaced by (35)-(36), defined from underlying polytopal meshes $\left(\mathfrak{T}_{m}\right)_{m \in \mathbb{N}}$ such that all the faces $\sigma \in \mathcal{F}_{m}$ are convex. Assume that $\left(\eta_{\mathfrak{T}_{m}}\right)_{m \in \mathbb{N}}$ is bounded (see (11)), and that $h_{\mathcal{M}_{m}} \rightarrow 0$ as $m \rightarrow \infty$.

Then the sequence $\left(\mathcal{D}_{m}\right)_{m \in \mathbb{N}}$ is coercive, GD-consistent, limit-conforming and compact in the sense of Definitions 2.2, 2.3, 2.4 and 2.5.

\section{Proof.}

- The coercivity property results from Lemma A.7 and from the equivalence of norms stated in Lemma 4.2 .

- The compactness property uses in addition Lemma A.6, as in the proof of Lemmas 3.12 and 3.13.

- Lemma 3.14 holds, by replacing the pointwise jump by the average jump.

- Replacing the pointwise jump by the average jump in Lemma 3.15, the whole proof holds except that, instead of $T_{3}=0$, we find

$$
\begin{aligned}
T_{3}=\sum_{K \in \mathcal{M}} \sum_{\sigma \in \mathcal{F}_{K}} \int_{\sigma}\left(\Pi_{\bar{K}} v(\boldsymbol{y})-v_{K, \sigma}^{a}\right) \boldsymbol{\varphi}(\boldsymbol{y}) \cdot \boldsymbol{n}_{K, \sigma} \mathrm{d} \gamma(\boldsymbol{y}) \\
=\sum_{K \in \mathcal{M}} \sum_{\sigma \in \mathcal{F}_{K}} \int_{\sigma}\left(\Pi_{\bar{K}} v(\boldsymbol{y})-v_{K, \sigma}^{a}\right)\left(\boldsymbol{\varphi}(\boldsymbol{y})-\pi_{K}^{0} \boldsymbol{\varphi}(\boldsymbol{y})\right) \cdot \boldsymbol{n}_{K, \sigma} \mathrm{d} \gamma(\boldsymbol{y})
\end{aligned}
$$

We then write, owing to Young's inequality,

$$
\left|T_{3}\right| \leq\left(\sum_{K \in \mathcal{M}} \sum_{\sigma \in \mathcal{F}_{K}} \frac{1}{d_{K, \sigma}^{p-1}} \int_{\sigma}\left|\Pi_{\bar{K}} v(\boldsymbol{y})-v_{K, \sigma}^{a}\right|^{p} \mathrm{~d} \gamma(\boldsymbol{y})\right)^{\frac{1}{p}}\left(\sum_{K \in \mathcal{M}} \sum_{\sigma \in \mathcal{F}_{K}} d_{K, \sigma}\left\|\boldsymbol{\varphi}-\pi_{K}^{0} \boldsymbol{\varphi}\right\|_{L^{p^{\prime}(\sigma)^{d}}}^{p^{\prime}}\right)^{\frac{1}{p^{\prime}}}
$$

Similarly again to (26), using $d_{K, \sigma} \leq h_{K}$, we can write

$$
\left\|\boldsymbol{\varphi}-\pi_{K}^{0} \boldsymbol{\varphi}\right\|_{L^{p^{\prime}(\sigma)^{d}}} \leq C h_{K}^{1-1 / p^{\prime}}\|\boldsymbol{\varphi}\|_{W^{1, p^{\prime}(K)^{d}}} \leq C h_{\mathcal{M}} d_{K, \sigma}^{-1 / p^{\prime}}\|\boldsymbol{\varphi}\|_{W^{1, p^{\prime}(K)^{d}}},
$$

where $C$ only depends on $\Omega, p, d$ and $\varrho$. The two preceding inequalities, in addition with inequality (37) in Lemma 4.1, allow to conclude (29) with $\ell=1$, which is sufficient for the limit conformity of the method. Note that we can nevertheless let $k \geq 1$ in the definition of the ADGGD, but this does not increase the order in the conclusion of Lemma 3.15 which remains equal to $\ell=1$.

\section{Application to the $p$-Laplace problem}

This section shows, on the example of the $p$-Laplace equation, the interest of plugging DG methods in the GDM framework. 


\subsection{Convergence and error estimate}

We consider the following problem.

$$
-\operatorname{div}\left(|\nabla \bar{u}|^{p-2} \nabla \bar{u}\right)=f+\operatorname{div}(\boldsymbol{F}) \text { in } \Omega,
$$

with boundary conditions

$$
\bar{u}=0 \text { on } \partial \Omega,
$$

under the following assumption, in addition to $p \in(1,+\infty)$ and $(1)$ :

$$
f \in L^{p^{\prime}}(\Omega), \boldsymbol{F} \in L^{p^{\prime}}(\Omega)^{d} .
$$

Under these hypotheses, the weak solution of (40) is the unique function $\bar{u}$ satisfying:

$$
\begin{aligned}
& \bar{u} \in W_{0}^{1, p}(\Omega) \text { and, for all } v \in W_{0}^{1, p}(\Omega), \\
& \int_{\Omega}|\nabla \bar{u}|^{p-2} \nabla \bar{u}(\boldsymbol{x}) \cdot \nabla v(\boldsymbol{x}) \mathrm{d} \boldsymbol{x}=\int_{\Omega} f(\boldsymbol{x}) v(\boldsymbol{x}) \mathrm{d} \boldsymbol{x}-\int_{\Omega} \boldsymbol{F}(\boldsymbol{x}) \cdot \nabla v(\boldsymbol{x}) \mathrm{d} \boldsymbol{x} .
\end{aligned}
$$

Definition 5.1 (Gradient scheme for the $p$-Laplace problem): Let $\mathcal{D}=\left(X_{\mathcal{D}, 0}, \Pi_{\mathcal{D}}, \nabla_{\mathcal{D}}\right)$ be a Gradient Discretisation in the sense of Definition 2.1. The corresponding gradient scheme for Problem (42) is defined by

$$
\begin{aligned}
& \text { Find } u \in X_{\mathcal{D}, 0} \text { such that, for any } v \in X_{\mathcal{D}, 0}, \\
& \int_{\Omega}\left|\nabla_{\mathcal{D}} u(\boldsymbol{x})\right|^{p-2} \nabla_{\mathcal{D}} u(\boldsymbol{x}) \cdot \nabla_{\mathcal{D}} v(\boldsymbol{x}) \mathrm{d} \boldsymbol{x}=\int_{\Omega} f(\boldsymbol{x}) \Pi_{\mathcal{D}} v(\boldsymbol{x}) \mathrm{d} \boldsymbol{x}-\int_{\Omega} \boldsymbol{F}(\boldsymbol{x}) \cdot \nabla_{\mathcal{D}} v(\boldsymbol{x}) \mathrm{d} \boldsymbol{x} .
\end{aligned}
$$

Remark 5.2: Either the DGGD scheme or the ADGGD scheme can be selected for defining the discrete operators used in (43). Note that, for any $v \in X_{\mathcal{D}, 0}$, the discrete gradient $\nabla_{\mathcal{D}} v$ defined by the DGGD scheme (15)-(16) is piecewise polynomial, even for $k=1$ in the case $d>1$. In this case, the computation of the left hand side of (43) implies the use of quadrature methods, which can be exact if $p=2$ or approximate in the general case. On the contrary, using the ADGGD scheme with $k=1$, the discrete gradient $\nabla_{\mathcal{D}} v$ defined by (35)-(36) is piecewise constant, and the computations are much easier (see the numerical examples in Section 5.3).

The following lemma, which is a consequence of the underlying minimisation problems and is proved in [9], establishes the existence and uniqueness of the solutions to (42) and (43), as well as estimates on these solutions.

Lemma 5.3: Under Hypotheses (41), there exists one and only one solution to each of the problems (42) and (43). These solutions moreover satisfy

$$
\|\nabla \bar{u}\|_{L^{p}(\Omega)^{d}} \leq\left(C_{P, p}\|f\|_{L^{p^{\prime}(\Omega)}}+\|\boldsymbol{F}\|_{L^{p^{\prime}(\Omega)^{d}}}\right)^{\frac{1}{p-1}}
$$

and

$$
\left\|\nabla_{\mathcal{D}} u_{\mathcal{D}}\right\|_{L^{p}(\Omega)^{d}} \leq\left(C_{\mathcal{D}}\|f\|_{L^{p^{\prime}(\Omega)}}+\|\boldsymbol{F}\|_{L^{p^{\prime}}(\Omega)^{d}}\right)^{\frac{1}{p-1}}
$$

where $C_{P, p}$ is the continuous Poincaré's constant in $W_{0}^{1, p}(\Omega)$, and $C_{\mathcal{D}}$ is defined by (2).

Theorem 5.4 (Control of the approximation error): Under Hypotheses (41), let $\bar{u} \in W_{0}^{1, p}(\Omega)$ be the solution of Problem (42), let $\mathcal{D}$ be a GD in the sense of Definition 2.1, and let $u_{\mathcal{D}} \in X_{\mathcal{D}, 0}$ be the solution to the gradient scheme (43). Then there exists $C_{4}>0$, depending only on $p$ such that:

1. If $p \in(1,2]$,

$$
\begin{aligned}
\left\|\nabla \bar{u}-\nabla_{\mathcal{D}} u_{\mathcal{D}}\right\|_{L^{p}(\Omega)^{d}} \leq S_{\mathcal{D}}(\bar{u}) & +C_{4}\left[W_{\mathcal{D}}\left(|\nabla \bar{u}|^{p-2} \nabla \bar{u}+\boldsymbol{F}\right)+S_{\mathcal{D}}(\bar{u})^{p-1}\right] \\
& \times\left[S_{\mathcal{D}}(\bar{u})^{p}+\left[\left(C_{\mathcal{D}}+C_{P, p}\right)\|f\|_{L^{p^{\prime}}(\Omega)}+\|\boldsymbol{F}\|_{L^{p^{\prime}(\Omega)^{d}}}\right]^{\frac{p}{p-1}}\right]^{\frac{2-p}{2}} .
\end{aligned}
$$


2. If $p \in(2,+\infty)$,

$$
\begin{aligned}
\left\|\nabla \bar{u}-\nabla_{\mathcal{D}} u_{\mathcal{D}}\right\|_{L^{p}(\Omega)^{d}} \leq S_{\mathcal{D}}(\bar{u}) & +C_{4}\left[W_{\mathcal{D}}\left(|\nabla \bar{u}|^{p-2} \nabla \bar{u}+\boldsymbol{F}\right)\right. \\
& \left.+S_{\mathcal{D}}(\bar{u})\left[\left(C_{P, p}\|f\|_{L^{p^{\prime}}(\Omega)}+\|\boldsymbol{F}\|_{L^{p^{\prime}}(\Omega)^{d}}\right)^{\frac{1}{p-1}}+S_{\mathcal{D}}(\bar{u})\right]^{p-2}\right]^{\frac{1}{p-1}} .
\end{aligned}
$$

As a consequence of (46)-(47), we have the following error estimate:

$$
\left\|\bar{u}-\Pi_{\mathcal{D}} u_{\mathcal{D}}\right\|_{L^{p}(\Omega)} \leq S_{\mathcal{D}}(\bar{u})+C_{\mathcal{D}}\left(S_{\mathcal{D}}(\bar{u})+\left\|\nabla \bar{u}-\nabla_{\mathcal{D}} u_{\mathcal{D}}\right\|_{L^{p}(\Omega)^{d}}\right) .
$$

The application of the previous theorem and of Lemmas proved in Section 3.2 allows to state and prove the following corollaries.

Corollary 5.5 (Convergence of schemes issued from DGGD or ADGGD for the $p$-Laplace problem):

Under Hypotheses (41), let $(\mathcal{D})_{m \in \mathbb{N}}$ be a sequence of DGGDs (or ADGGDs) such that $h_{\mathfrak{T}_{m}}$ tends to 0 as $m \rightarrow \infty$ while $\eta_{\mathfrak{E}_{m}}$ remains bounded (the faces of the meshes being convex in the case of ADGGDs). Let $\bar{u}$ and $u_{\mathcal{D}_{m}}$ be the respective solution to Problems (42) and (43) for all $m \in \mathbb{N}$. Then $\Pi_{\mathcal{D}_{m}} u_{\mathcal{D}_{m}}$ (resp. $\nabla_{\mathcal{D}_{m}} u_{\mathcal{D}_{m}}$ ) converge to $\bar{u}$ in $L^{p}(\Omega)$ (resp. to $\nabla \bar{u}$ in $\left.L^{p}(\Omega)^{p}\right)$ as $m \rightarrow \infty$.

Proof. Owing to Theorem 3.11 (for DGGDs) or 4.4 (for ADGGDs), $C_{\mathcal{D}_{m}}$ remains bounded, whereas $S_{\mathcal{D}_{m}}(\bar{u})$ and $W_{\mathcal{D}_{m}}\left(|\nabla \bar{u}|^{p-2} \nabla \bar{u}+\boldsymbol{F}\right)$ tend to 0 as $m \rightarrow \infty$. Hence (46) or (47) on one hand, and (48) on the other hand, show the conclusion of the corollary.

Corollary 5.6 (Error estimate of the discontinuous Galerkin methods for the $p$-Laplace problem):

Under Hypotheses (41), let us assume that $p \in(1,2], \Omega=(0,1), f \in L^{\infty}(\Omega)$ and $\boldsymbol{F}(x)=0$ for all $x \in \Omega$. Let $\mathcal{D}$ be a DGGD (in this case identical to ADGGD) in the sense of Definition 3.2 where the polytopal mesh of $\Omega$ is uniform. Let $\bar{u}$ and $u_{\mathcal{D}}$ be the respective solution to Problems (42) and (43). Then there holds

$$
\left\|\bar{u}-\Pi_{\mathcal{D}} u_{\mathcal{D}}\right\|_{L^{p}(\Omega)}+\left\|\nabla \bar{u}-\nabla_{\mathcal{D}} u_{\mathcal{D}}\right\|_{L^{p}(\Omega)} \leq C h_{\mathcal{M}}^{p-1}
$$

where $C$ only depends on $f, \beta, p$ and $k$.

Proof. In this particular case, the continuous solution $\bar{u}$ satisfies that there exists $C \in \mathbb{R}$ with

$$
\forall x \in[0,1],\left|\bar{u}^{\prime}(x)\right|^{p-2} \bar{u}^{\prime}(x)=C-\int_{0}^{x} f(s) \mathrm{d} s,
$$

and therefore

$$
\forall x \in[0,1], \bar{u}^{\prime}(x)=\left|C-\int_{0}^{x} f(s) \mathrm{d} s\right|^{\frac{1}{p-1}} \operatorname{sign}\left(C-\int_{0}^{x} f(s) \mathrm{d} s\right) .
$$

Then, since $\frac{1}{p-1}>1$, we get that $\bar{u} \in W_{0}^{1, p}(\Omega) \cap W^{2, \infty}(\Omega)$ and $\left|\bar{u}^{\prime}\right|^{p-2} \bar{u}^{\prime} \in W^{1, \infty}(\mathbb{R})$ (extending $f$ by 0 outside $\Omega$ ). Therefore we apply Lemmas 3.12, 3.14 and 3.15, which, in addition to (46), complete the proof of (49).

Note that this error estimate provides, in the case $p=2$, the order 1 for the error estimate of the derivative of the solution, which is confirmed by the numerical results below. But it does not provide the order 2 observed on the error estimate of the solution.

\subsection{Numerical results in the one-dimensional case}

We consider Problem (40a) where $d=1, \Omega=(0,1), f(x)=1$ and $\boldsymbol{F}(x)=0$. The analytical solution is then given by

$$
\bar{u}(x)=\frac{p-1}{p}\left[\left(\frac{1}{2}\right)^{p /(p-1)}-\left|x-\frac{1}{2}\right|^{p /(p-1)}\right] .
$$

We consider a mesh with constant space step $h=1 / N$ (with $N \in \mathbb{N}^{\star}$ ) and we use Scheme (43) together with the Discontinuous Galerkin gradient discretisation given by Definition 3.2, with $k=1$ and $\beta=1 / 2$ 


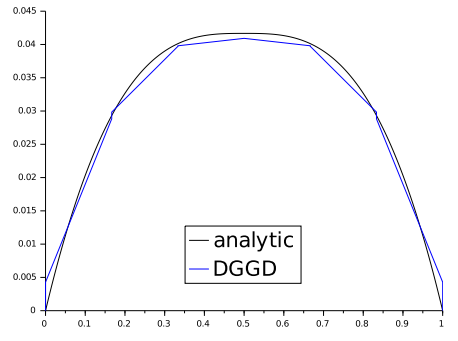

(a) $p=1.5$

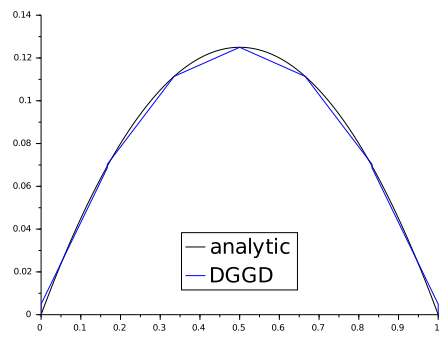

(b) $p=2$

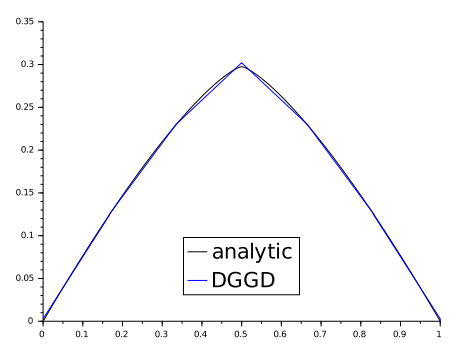

(c) $p=4$

Fig. 2: Exact and DGGD approximate solutions for the $p$-Laplace equation $(k=1, \beta=0.5$, $N=6)$.

(note that in the one-dimensional case, the two definitions (15) and (35) for the discrete gradient are identical, so the DGGD is identical to the ADGGD).

We see in Figure 2 that the approximate solution matches quite well the analytical solution for $N=6$, considering the three cases $p=1.5, p=2$ and $p=4$. The convergence orders which are given in Table 1, are higher than that which could be expected from Corollary 5.6 in the case $p \leq 2$.

\begin{tabular}{|c|c|c||c|c||c|c|}
\hline & \multicolumn{2}{|c||}{$p=1.5$} & \multicolumn{2}{c||}{$p=2$} & \multicolumn{2}{c|}{$p=4$} \\
\hline & $u$ & $\nabla u$ & $u$ & $\nabla u$ & $u$ & $\nabla u$ \\
\hline$N=10$ & $5.51 \mathrm{E}-04$ & $6.34 \mathrm{E}-03$ & $9.65 \mathrm{E}-04$ & $9.40 \mathrm{E}-03$ & $1.48 \mathrm{E}-03$ & $8.11 \mathrm{E}-03$ \\
\hline order & 1.85 & 1.55 & 1.96 & 1.50 & 1.62 & 1.33 \\
\hline$N=20$ & $1.53 \mathrm{E}-04$ & $2.17 \mathrm{E}-03$ & $2.48 \mathrm{E}-04$ & $3.32 \mathrm{E}-03$ & $4.80 \mathrm{E}-04$ & $3.23 \mathrm{E}-03$ \\
\hline order & 1.92 & 1.61 & 1.98 & 1.50 & 1.60 & 1.29 \\
\hline$N=40$ & $4.02 \mathrm{E}-05$ & $7.11 \mathrm{E}-04$ & $6.29 \mathrm{E}-05$ & $1.18 \mathrm{E}-03$ & $1.58 \mathrm{E}-04$ & $1.33 \mathrm{E}-03$ \\
\hline order & 1.96 & 1.64 & 1.99 & 1.50 & 1.59 & 1.59 \\
\hline$N=80$ & $1.03 \mathrm{E}-05$ & $2.28 \mathrm{E}-04$ & $1.58 \mathrm{E}-05$ & $4.15 \mathrm{E}-04$ & $5.24 \mathrm{E}-05$ & $5.51 \mathrm{E}-04$ \\
\hline order & 1.98 & 1.65 & 2.00 & 1.50 & 1.59 & 1.26 \\
\hline$N=160$ & $2.62 \mathrm{E}-06$ & $7.26 \mathrm{E}-05$ & $3.97 \mathrm{E}-06$ & $3.97 \mathrm{E}-06$ & $1.74 \mathrm{E}-05$ & $2.30 \mathrm{E}-04$ \\
\hline
\end{tabular}

Tab. 1: Errors and rates of convergences, on the functions and the gradient, for the ADGGD scheme applied to the $p$-Laplace equation in dimension 1. "Order" represents the rate of convergence from the line above to the line below. 


\subsection{Numerical results in the two-dimensional case}

We consider Problem (40a) with $d=2, \Omega=(0,1) \times(0,1), f(\boldsymbol{x})=2$ and $\boldsymbol{F}(x)=0$ for all $\boldsymbol{x} \in \Omega$. We define $\boldsymbol{x}_{\Omega}=(1 / 2,1 / 2)$ and we prescribe non-homogeneous Dirichlet boundary conditions in agreement with the analytical solution

$$
\bar{u}(\boldsymbol{x})=\frac{p-1}{p}\left[\left(\frac{1}{\sqrt{2}}\right)^{p /(p-1)}-\left|\boldsymbol{x}-\boldsymbol{x}_{\Omega}\right|^{p /(p-1)}\right] .
$$

We apply Scheme (43) together with the Average Discontinuous Galerkin Gradient Discretisation given by Definition 3.2 and definition (35) for the discrete gradient, letting $k=1$ and $\beta=4 / 5$. The triangular meshes from the family mesh1 of [14] are used for the numerical tests.

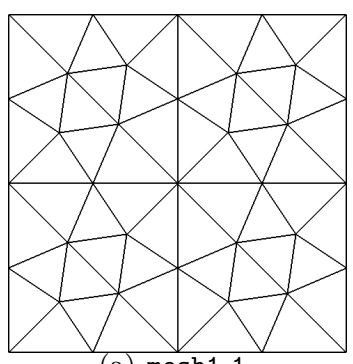

(a) mesh1_1

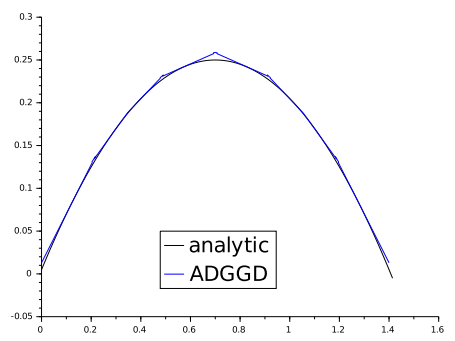

(c) $p=2$

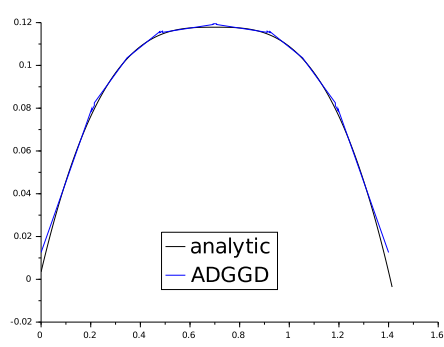

(b) $p=1.5$

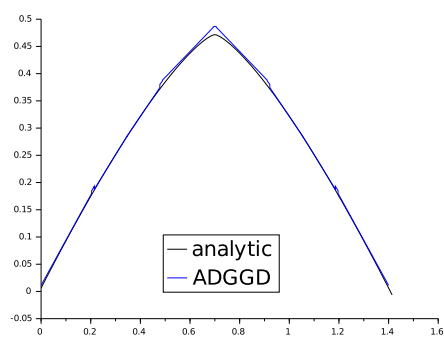

(d) $p=4$

Fig. 3: Mesh mesh1_1 and exact and ADGGD approximate profiles along the line $x_{2}=x_{1}+0.01$ for the $p$-Laplace equation $(k=1, \beta=0.8$, using mesh1_1).

Figure 3 presents the profile of the approximate solution along the line $x_{2}=x_{1}+0.01$, for the three cases $p=1.5, p=2$ and $p=4$, on the coarsest triangular mesh. We notice a rather good match of approximate solution on this line.

Table 2 shows that the practical rates of convergence are better than the theoretical ones from Theorem 5.4; however, the rates for the gradient are degraded with respect to the similar test case in dimension $d=1$.

\section{Application to the heterogeneous Dirichlet problem}

\subsection{Link with the SIPG scheme}

Let us prove that the gradient scheme issued from the DGGD defined in Section 3 is identical to the Symmetric Interior Penalty Galerkin (SIPG) method $[1,2,10]$ for the following elliptic problem:

$$
\bar{u} \in H_{0}^{1}(\Omega), \forall \bar{v} \in H_{0}^{1}(\Omega), \int_{\Omega} \Lambda(\boldsymbol{x}) \nabla \bar{u}(\boldsymbol{x}) \cdot \nabla \bar{v}(\boldsymbol{x}) \mathrm{d} \boldsymbol{x}=\int_{\Omega} f(\boldsymbol{x}) \bar{v}(\boldsymbol{x}) \mathrm{d} \boldsymbol{x},
$$




\begin{tabular}{|c|c|c||c|c||c|c|}
\hline & \multicolumn{2}{|c||}{$p=1.5$} & \multicolumn{2}{c||}{$p=2$} & \multicolumn{2}{c|}{$p=4$} \\
\hline & $u$ & $\nabla u$ & $u$ & $\nabla u$ & $u$ & $\nabla u$ \\
\hline mesh1_1 & $0.944 \mathrm{E}-03$ & $0.314 \mathrm{E}-02$ & $0.120 \mathrm{E}-02$ & $0.423 \mathrm{E}-02$ & $0.138 \mathrm{E}-02$ & $0.432 \mathrm{E}-02$ \\
\hline order & 1.96 & 1.48 & 1.95 & 1.42 & 1.32 & 1.41 \\
\hline mesh1_2 & $0.243 \mathrm{E}-03$ & $0.113 \mathrm{E}-02$ & $0.308 \mathrm{E}-03$ & $0.158 \mathrm{E}-02$ & $0.555 \mathrm{E}-03$ & $0.162 \mathrm{E}-02$ \\
\hline order & 1.97 & 1.48 & 1.98 & 1.38 & 1.57 & 1.16 \\
\hline mesh1_3 & $0.621 \mathrm{E}-04$ & $0.405 \mathrm{E}-03$ & $0.783 \mathrm{E}-04$ & $0.608 \mathrm{E}-03$ & $0.187 \mathrm{E}-03$ & $0.727 \mathrm{E}-03$ \\
\hline order & 1.98 & 1.40 & 1.99 & 1.31 & 1.67 & 0.93 \\
\hline mesh1_4 & $0.157 \mathrm{E}-04$ & $0.154 \mathrm{E}-03$ & $0.197 \mathrm{E}-04$ & $0.245 \mathrm{E}-03$ & $0.587 \mathrm{E}-04$ & $0.381 \mathrm{E}-03$ \\
\hline order & 1.99 & 1.29 & 1.99 & 1.23 & 1.73 & 0.85 \\
\hline mesh1_5 & $0.396 \mathrm{E}-05$ & $0.630 \mathrm{E}-04$ & $0.495 \mathrm{E}-05$ & $0.105 \mathrm{E}-03$ & $0.177 \mathrm{E}-04$ & $0.211 \mathrm{E}-03$ \\
\hline
\end{tabular}

Tab. 2: Errors and rates of convergences, on the functions and the gradient, for the ADGGD scheme applied to the $p$-Laplace equation in dimension 2. "Order" represents the rate of convergence from the line above to the line below.

where the assumptions are:

- $\Lambda$ is a measurable function from $\Omega$ to the set of $d \times d$

symmetric matrices and there exists $\underline{\lambda}, \bar{\lambda}>0$ such that,

for a.e. $\boldsymbol{x} \in \Omega, \Lambda(\boldsymbol{x})$ has eigenvalues in $[\underline{\lambda}, \bar{\lambda}]$,

- $f \in L^{2}(\Omega)$.

Using the DGGD $\mathcal{D}=\left(X_{\mathcal{D}, 0}, \Pi_{\mathcal{D}}, \nabla_{\mathcal{D}}\right)$ in the sense of Definition 3.2, the gradient scheme for the discretisation of (52) is given by:

$$
u \in X_{\mathcal{D}, 0}, \forall v \in X_{\mathcal{D}, 0}, \int_{\Omega} \Lambda(\boldsymbol{x}) \nabla_{\mathcal{D}} u(\boldsymbol{x}) \cdot \nabla_{\mathcal{D}} v(\boldsymbol{x}) \mathrm{d} \boldsymbol{x}=\int_{\Omega} f(\boldsymbol{x}) \Pi_{\mathcal{D}} v(\boldsymbol{x}) \mathrm{d} \boldsymbol{x} .
$$

It is proved in [12, Lemma 2.2] that the solution $u$ to (54) satisfies

$$
\begin{aligned}
& \left\|\nabla \bar{u}-\nabla_{\mathcal{D}} u\right\|_{L^{2}(\Omega)} \leq \frac{1}{\underline{\lambda}}\left[W_{\mathcal{D}}(\Lambda \nabla \bar{u})+(\bar{\lambda}+\underline{\lambda}) S_{\mathcal{D}}(\bar{u})\right] \\
& \left\|\bar{u}-\Pi_{\mathcal{D}} u\right\|_{L^{2}(\Omega)} \leq \frac{1}{\underline{\lambda}}\left[C_{\mathcal{D}} W_{\mathcal{D}}(\Lambda \nabla \bar{u})+\left(C_{\mathcal{D}} \bar{\lambda}+\underline{\lambda}\right) S_{\mathcal{D}}(\bar{u})\right]
\end{aligned}
$$

where $C_{\mathcal{D}}, S_{\mathcal{D}}$ and $W_{\mathcal{D}}$ are defined in Section 2. The error estimate (55)-(56) together with Lemma 3.12 for a bound on $C_{\mathcal{D}}$, estimates $(23)$ on $S_{\mathcal{D}}(\bar{u})$ and $(29)$ on $W_{\mathcal{D}}(\Lambda \nabla \bar{u})$ yield the high order error estimate of the method.

Let us assume that $\Lambda$ restricted to $K \in \mathcal{M}$ is constant in $K$ (this is not needed for the DGGD scheme, but it is necessary for defining the SIPG scheme in a heterogeneous case (see [8, Assumption 4.43]). Then the left hand side of (54) can be developed in this particular case, using the change the variable $\boldsymbol{x} \mapsto(\boldsymbol{y}=\boldsymbol{Y}(\boldsymbol{x}), s=S(\boldsymbol{x}))$. Indeed, we first write

$$
\begin{array}{r}
\int_{D_{K, \sigma}} \Lambda(\boldsymbol{x}) \psi(S(\boldsymbol{x})) \frac{[u]_{K, \sigma}(\boldsymbol{Y}(\boldsymbol{x}))}{d_{K, \sigma}} \boldsymbol{n}_{K, \sigma} \cdot \nabla_{\bar{K}} v(\boldsymbol{x}) \mathrm{d} \boldsymbol{x} \\
=\int_{\sigma} \Lambda_{K} \frac{[u]_{K, \sigma}(\boldsymbol{y})}{d_{K, \sigma}} \boldsymbol{n}_{K, \sigma} \cdot \int_{\beta}^{1} \nabla_{\bar{K}} v(\boldsymbol{x}(\boldsymbol{y}, s)) \psi(s) s^{d-1} \mathrm{~d} s \mathrm{~d} \gamma(\boldsymbol{y}) d_{K, \sigma} .
\end{array}
$$

Let us now remark that the function $\varphi(s)=\nabla_{\bar{K}} v\left(\boldsymbol{x}_{K}+s\left(\boldsymbol{y}-\boldsymbol{x}_{K}\right)\right) \cdot \boldsymbol{n}_{K, \sigma}$ is such that

$$
\varphi(s)=\nabla_{\bar{K}} v(\boldsymbol{y}) \cdot \boldsymbol{n}_{K, \sigma}+\sum_{m=1}^{k-1} p_{m}(\boldsymbol{y})(1-s)^{m},
$$


where, for $m=1, \ldots, k-1, p_{m}(\boldsymbol{y})$ is a polynomial with degree less or equal to $k-1$ with respect to the coordinates of $\boldsymbol{y}$. Therefore, (17)-(18) imply that $\int_{\beta}^{1} \nabla_{\bar{K}} v(\boldsymbol{x}(\boldsymbol{y}, s)) \cdot \boldsymbol{n}_{K, \sigma} \psi(s) s^{d-1} \mathrm{~d} s=\nabla_{\bar{K}} v(\boldsymbol{y}) \cdot \boldsymbol{n}_{K, \sigma}$. Hence we get

$$
\begin{array}{r}
\int_{D_{K, \sigma}} \Lambda(\boldsymbol{x}) \psi(S(\boldsymbol{x})) \frac{[u]_{K, \sigma}(\boldsymbol{Y}(\boldsymbol{x}))}{d_{K, \sigma}} \boldsymbol{n}_{K, \sigma} \cdot \nabla_{\bar{K}} v(\boldsymbol{x}) \mathrm{d} \boldsymbol{x} \\
=\int_{\sigma} \Lambda_{K}[u]_{K, \sigma}(\boldsymbol{y}) \boldsymbol{n}_{K, \sigma} \cdot \nabla_{\bar{K}} v(\boldsymbol{y}) \mathrm{d} \gamma(\boldsymbol{y})
\end{array}
$$

We also have

$$
\begin{aligned}
& \int_{D_{K, \sigma}} \Lambda(\boldsymbol{x}) \frac{[u]_{K, \sigma}(\boldsymbol{Y}(\boldsymbol{x}))}{d_{K, \sigma}} \boldsymbol{n}_{K, \sigma} \cdot \frac{[v]_{K, \sigma}(\boldsymbol{x})}{d_{K, \sigma}} \boldsymbol{n}_{K, \sigma} \psi(S(\boldsymbol{x}))^{2} \mathrm{~d} \boldsymbol{x} \\
= & \frac{\Lambda_{K} \boldsymbol{n}_{K, \sigma} \cdot \boldsymbol{n}_{K, \sigma}}{d_{K, \sigma}} \int_{\beta}^{1} \psi(s)^{2} s^{d-1} \mathrm{~d} s \int_{\sigma}[u]_{K, \sigma}(\boldsymbol{y})[v]_{K, \sigma}(\boldsymbol{y}) \mathrm{d} \gamma(\boldsymbol{y}) .
\end{aligned}
$$

Then the bilinear form involved in Gradient Scheme (54) satisfies

$$
\begin{aligned}
& \int_{\Omega} \Lambda(\boldsymbol{x}) \nabla_{\mathcal{D}} u(\boldsymbol{x}) \cdot \nabla_{\mathcal{D}} v(\boldsymbol{x}) \mathrm{d} \boldsymbol{x} \\
& =\sum_{K \in \mathcal{M}}\left(\int_{K} \Lambda_{K} \nabla_{\bar{K}} u(\boldsymbol{x}) \cdot \nabla_{\bar{K}} v(\boldsymbol{x}) \mathrm{d} \boldsymbol{x}\right. \\
& \quad+\sum_{\sigma \in \mathcal{F}_{K}}\left(\int_{\sigma} \Lambda_{K}\left([u]_{K, \sigma}(\boldsymbol{y}) \nabla_{\bar{K}} v(\boldsymbol{y})+[v]_{K, \sigma}(\boldsymbol{y}) \nabla_{\bar{K}} u(\boldsymbol{y})\right) \cdot \boldsymbol{n}_{K, \sigma} \mathrm{d} \gamma(\boldsymbol{y})\right. \\
& \left.\left.\quad+\frac{\Lambda_{K} \boldsymbol{n}_{K, \sigma} \cdot \boldsymbol{n}_{K, \sigma}}{d_{K, \sigma}} \int_{\beta}^{1} \psi(s)^{2} s^{d-1} \mathrm{~d} s \int_{\sigma}[u]_{K, \sigma}(\boldsymbol{y})[v]_{K, \sigma}(\boldsymbol{y}) \mathrm{d} \gamma(\boldsymbol{y})\right)\right) .
\end{aligned}
$$

We then recover the SIPG scheme as presented in [10] or [8], the penalty coefficient $\tau_{\sigma}\left(\right.$ term $\frac{\sigma_{e}}{|e|^{\beta_{0}}}$ of $[10$, eqn. (11)], term $\frac{\eta}{h_{F}}$ of $[8$, eqn. (4.12)]) being equal, in the preceding relation, to

$$
\tau_{\sigma}=\frac{1}{4} \int_{\beta}^{1} \psi(s)^{2} s^{d-1} \mathrm{~d} s\left(\frac{\Lambda_{K} \boldsymbol{n}_{K, \sigma} \cdot \boldsymbol{n}_{K, \sigma}}{d_{K, \sigma}}+\frac{\Lambda_{L} \boldsymbol{n}_{L, \sigma} \cdot \boldsymbol{n}_{L, \sigma}}{d_{L, \sigma}}\right) \text { if } \mathcal{M}_{\sigma}=\{K, L\},
$$

and

$$
\tau_{\sigma}=\int_{\beta}^{1} \psi(s)^{2} s^{d-1} \mathrm{~d} s \frac{\Lambda_{K} \boldsymbol{n}_{K, \sigma} \cdot \boldsymbol{n}_{K, \sigma}}{d_{K, \sigma}} \text { if } \mathcal{M}_{\sigma}=\{K\} .
$$

Note that, since the Cauchy-Schwarz inequality implies

$$
\left(\int_{\beta}^{1} \psi(s) s^{d-1} \mathrm{~d} s\right)^{2} \leq \int_{\beta}^{1} \psi(s)^{2} s^{d-1} \mathrm{~d} s \int_{\beta}^{1} s^{d-1} \mathrm{~d} s,
$$

we get that

$$
\int_{\beta}^{1} \psi(s)^{2} s^{d-1} \mathrm{~d} s \geq \frac{d}{1-\beta^{d}} \geq d,
$$

which provides a minimum value for $\tau_{\sigma}$ letting $\beta \rightarrow 0$ (such minimum values are given, for example, by [8, Lemma 4.12]. In our setting, it does not depend on the regularity of the mesh nor on the maximum cardinal of $\mathcal{F}_{K}$ (in the DGGD scheme, we don't handle separately the case $d=1$ and the cases $d>1$ ).

Remark 6.1: The above expressions prescribed for $\tau_{\sigma}$ involve the arithmetic averaging of the diffusion tensors in the cells $K$ and $L$. An interesting point to be studied is the numerical comparison on heterogeneous cases with $[11,5]$, where the authors introduce an expression involving the harmonic averaging of these diffusion tensors. 


\subsection{Numerical results}

The aim of this section is to assess the influence of the parameter $\beta \in(0,1)$ on the accuracy of the gradient scheme (54) issued from the DGGD for the discretisation of $(52)$. We consider the $1 D$ case $\Omega=(0,1)$, and the polytopal mesh $\mathfrak{T}$ defined, for $N \in \mathbb{N}^{\star}$ and $h=\frac{1}{N}$, by $\mathcal{M}=\{](i-1) h, i h[, i=1, \ldots, N\}$, $\mathcal{F}=\{\{i h\}, i=0, \ldots, N\}, \mathcal{P}=\left\{\left(i-\frac{1}{2}\right) h, i=1, \ldots, N\right\}$. We consider one of the test cases studied in [10], that is Problem (52) with $\Lambda=\operatorname{Id}$ and $\bar{u}(x)=\cos (8 \pi x)-1$ (hence $f(x)=(8 \pi)^{2} \cos (8 \pi x)$ ). Considering first degree polynomials, the set $X_{\mathcal{D}, 0}$ is a vector space with dimension $2 N$. In Table 3 , the columns "FE" correspond to the conforming $\mathbb{P}^{1}$ Finite Element solution, we check that the results provided by "[10]" with $\sigma_{n}=4.5$, which corresponds to $\beta=1-1 / \sigma_{n}$ for the interior faces, and $\beta=1-2 / \sigma_{n}$ for the exterior faces, are close to ours: Although we did not prove that the linear systems are invertible when $\beta=0$, we

\begin{tabular}{|c||c|c|c|c|c|c|}
\hline$N \backslash \beta$ & 0 & 0.5 & 0.9 & 0.99 & $\mathrm{FE}$ & {$[10]$} \\
\hline 10 & 0.496 & 0.241 & 0.347 & 0.394 & 0.399 & 0.247 \\
\hline order & 1.438 & 1.529 & 1.734 & 1.843 & 1.855 & \\
\hline 20 & 0.183 & 0.083 & 0.104 & 0.110 & 0.110 & 0.083 \\
\hline order & 1.092 & 1.706 & 1.909 & 1.959 & 1.964 & \\
\hline 40 & 0.086 & 0.026 & 0.028 & 0.028 & 0.028 & 0.024 \\
\hline order & 1.013 & 1.894 & 1.973 & 1.989 & 1.991 & \\
\hline 80 & 0.043 & 0.007 & 0.007 & 0.007 & 0.007 & \\
\hline order & 0.999 & 1.967 & 1.992 & 1.997 & 1.998 & \\
\hline 160 & 0.021 & 0.002 & 0.002 & 0.002 & 0.002 & \\
\hline \multicolumn{7}{|c|}{$L^{2}$ error of the solution } \\
\hline
\end{tabular}

\begin{tabular}{|c||c|c|c|c|c|c|}
\hline$N \backslash \beta$ & 0 & 0.5 & 0.9 & 0.99 & $\mathrm{FE}$ & {$[10]$} \\
\hline 10 & 13.233 & 11.533 & 11.360 & 11.349 & 11.348 & 11.777 \\
\hline order & 0.172 & 0.781 & 0.862 & 0.863 & 0.863 & \\
\hline 20 & 11.743 & 6.714 & 6.251 & 6.240 & 6.240 & 6.421 \\
\hline order & 0.010 & 1.004 & 0.966 & 0.965 & 0.965 & \\
\hline 40 & 11.666 & 3.348 & 3.199 & 3.197 & 3.197 & 3.253 \\
\hline order & -0.008 & 1.034 & 0.992 & 0.991 & 0.991 & \\
\hline 80 & 11.728 & 1.635 & 1.609 & 1.608 & 1.608 & \\
\hline order & -0.007 & 1.014 & 0.998 & 0.998 & 0.998 & \\
\hline 160 & 11.781 & 0.810 & 0.805 & 0.805 & 0.805 & \\
\hline \multicolumn{7}{|c|}{ error of the broken gradient } \\
\hline
\end{tabular}

Tab. 3: Errors and rates of convergences, on the functions and the gradient, for the DGGD scheme applied to Problem (52). "Order" represents the rate of convergence from the line above to the line below.

note that in practice a solution is obtained but that the broken gradient does not seem to converge. In this very regular case, the $L^{2}$ error is the lowest for $\beta=0.5$ but the convergence seems slightly better for $\beta$ closer to 1 , and it tends to the results of the finite element method as $\beta \rightarrow 1$.

\section{Conclusion}

The two versions of the DG method included in the GDM framework have the advantages to hold on any polytopal mesh provided that the grid cells are strictly star-shaped, to involve Discrete Functional Analysis results which do not depend on the regularity of the mesh, and to apply on any problem on which the GDM is shown to converge (like the example of the $p$-Laplace problem taken in this paper). The DGGD scheme, identical to the SIPG method in the case of linear diffusion problems with constant diffusion in the cells, applies to the case of any measurable diffusion field. In the first order case, the ADGGD scheme presents the advantage of yielding simple computations owing to piecewise constant discrete gradients.

\section{A Discrete functional analysis}

Since our geometric hypotheses are different from those done in $[7,8]$, we now provide the Sobolev inequalities suited to Definition 3.1 of polytopal meshes. The techniques of proof are identical to that of $[8$, Theorem 5.3]. In this section, we denote, for $\xi \in \mathbb{R}^{d}$ by $|\xi|=\left(\sum_{i=1}^{d} \xi_{i}^{2}\right)^{1 / 2}$ the Euclidean norm of $\xi$.

Lemma A.1: Let $k, d \in \mathbb{N}^{\star}, \beta \in(0,1)$ and let $\psi:(0,1) \rightarrow \mathbb{R}$ be the function such that $\psi(s)=0$ on $(0, \beta)$, $\psi_{\mid[\beta, 1]} \in \mathbb{P}_{k-1}([\beta, 1])$ and that (17)-(18) hold. Then there exists $C>0$, only depending on $d$ and $k$, such that

$$
\frac{d}{1-\beta^{d}} \leq\|\psi\|_{\infty} \leq \frac{C}{1-\beta},
$$

denoting for short by $\|\psi\|_{\infty}$ the $L^{\infty}((0,1))$ norm of $\psi$. 
Proof. Thanks to (17), there holds

$$
1=\int_{\beta}^{1} \psi(s) s^{d-1} \mathrm{~d} s \leq\|\psi\|_{\infty} \int_{\beta}^{1} s^{d-1} \mathrm{~d} s=\|\psi\|_{\infty} \frac{1-\beta^{d}}{d},
$$

which proves the left inequality of (57). Writing, for $s \in(\beta, 1), \psi(s)=\sum_{i=0}^{k-1} u_{i}(1-s)^{i}$, multiplying (17) by $u_{0}$ and (18) by $u_{i}$, summing all these equations for $i=1, \ldots, k-1$, we get

$$
\int_{\beta}^{1}\left(\sum_{i=0}^{k-1} u_{i}(1-s)^{i}\right)^{2} s^{d-1} \mathrm{~d} s=u_{0} .
$$

Making the change of variable $t=(1-s) /(1-\beta)$, we get

$$
\int_{0}^{1}\left(\sum_{i=0}^{k-1} u_{i}(1-\beta)^{i} t^{i}\right)^{2}(1-t(1-\beta))^{d-1}(1-\beta) \mathrm{d} t=u_{0} .
$$

Owing to $1-t(1-\beta) \geq 1-t$, we can write

$$
(1-\beta) \int_{0}^{1}\left(\sum_{i=0}^{k-1} u_{i}(1-\beta)^{i} t^{i}\right)^{2}(1-t)^{d-1} \mathrm{~d} t \leq u_{0}
$$

Let $\alpha_{k, d}>0$ be the lowest eigenvalue of the Gram matrix $M$ defined by $M_{i j}=\int_{0}^{1} t^{i+j-2}(1-t)^{d-1} \mathrm{~d} t$, $i, j=1, \ldots, k$. This value is such that

$$
\forall\left(v_{0}, \ldots, v_{k-1}\right) \in \mathbb{R}^{k}, \alpha_{k, d} \sum_{i=0}^{k-1} v_{i}^{2} \leq \int_{0}^{1}\left(\sum_{i=0}^{k-1} v_{i} t^{i}\right)^{2}(1-t)^{d-1} \mathrm{~d} t .
$$

We then have, letting $v_{i}=u_{i}(1-\beta)^{i}$ for $i=0, \ldots, k-1$,

$$
(1-\beta) \alpha_{k, d}\left(u_{0}^{2}+\sum_{i=1}^{k-1}\left(u_{i}(1-\beta)^{i}\right)^{2}\right) \leq u_{0}
$$

yielding on one hand $u_{0} \leq \frac{1}{(1-\beta) \alpha_{k, d}}$ and therefore on the other hand

$$
\left(\sum_{i=0}^{k-1}\left(u_{i}(1-\beta)^{i}\right)^{2}\right)^{1 / 2} \leq \frac{1}{(1-\beta) \alpha_{k, d}}
$$

We then get, using the Cauchy-Schwarz inequality, that

$$
\forall s \in(\beta, 1),|\psi(s)| \leq \sum_{i=0}^{k-1}\left|u_{i}\right|(1-\beta)^{i} \leq \sqrt{k}\left(\sum_{i=0}^{k-1}\left(u_{i}(1-\beta)^{i}\right)^{2}\right)^{1 / 2} \leq \frac{\sqrt{k}}{(1-\beta) \alpha_{k, d}},
$$

hence leading to the right inequality of (57).

The constant $C_{q, n}>0$, introduced by the following definition, is used in the course of this mathematical study for $(q, n)=(p, k-1)$, and $(q, n)=\left(\frac{p d}{d-p}, k\right)$ when $\left.p \in\right] 1, d[$.

Definition A.2 (Comparison of norms on $\mathbb{R}^{n+1}$ ): Let $d \in \mathbb{N}^{\star}, n \in \mathbb{N}$ and $q>0$ be given. We denote by $C_{q, n}>0$ the greatest constant, depending only on $n, q$ and $d$, such that

$$
\forall\left(a_{0}, \ldots, a_{n}\right) \in \mathbb{R}^{n+1}, \int_{0}^{1}\left|\sum_{i=0}^{n} a_{i} s^{i}\right|{ }^{q} s^{d-1} \mathrm{~d} s \geq C_{q, n} \sum_{i=0}^{n}\left|a_{i}\right|^{q} .
$$

The following lemma plays an essential role in the study of $\left\|\nabla_{\mathcal{D}} \cdot\right\|_{L^{p}(\Omega)}$. 
Lemma A.3: Let $n \in \mathbb{N}$ and $\beta \in(0,1)$ be given. Let $\mathfrak{T}$ be a polytopal mesh in the sense of Definition 3.1 . Then there holds

$$
\begin{array}{r}
\forall v \in \mathbb{P}_{n}\left(\mathbb{R}^{d}\right), \forall K \in \mathcal{M}, \forall \sigma \in \mathcal{F}_{K}, \\
\int_{D_{K, \sigma}}|v(\boldsymbol{x})|^{p} \mathrm{~d} \boldsymbol{x} \leq \frac{(n+1)^{p-1}}{\beta^{d+p n} C_{p, n}} \int_{D_{K, \sigma}^{(\beta)}}|v(\boldsymbol{x})|^{p} \mathrm{~d} \boldsymbol{x},
\end{array}
$$

where $C_{p, n}$ is defined in Definition A.2 with $q=p$, and where $D_{K, \sigma}^{(\beta)}$ is defined by (19).

Proof. For $K \in \mathcal{M}$ and $\sigma \in \mathcal{F}_{K}$, we compute $\int_{D_{K, \sigma}^{(\beta)}}|v(\boldsymbol{x})|^{p} \mathrm{~d} \boldsymbol{x}$ with making the change of variable $\boldsymbol{x} \mapsto(\boldsymbol{y}=\boldsymbol{Y}(\boldsymbol{x}), s=S(\boldsymbol{x}))$, where $\boldsymbol{y} \in \sigma$ and $s \in(0, \beta)$. We then have $\mathrm{d} \boldsymbol{x}=d_{K, \sigma} s^{d-1} \mathrm{~d} \gamma(\boldsymbol{y}) \mathrm{d} s$, which leads to

$$
\int_{D_{K, \sigma}^{(\beta)}}|v(\boldsymbol{x})|^{p} \mathrm{~d} \boldsymbol{x}=\int_{\sigma} \int_{0}^{\beta}\left|v\left(\boldsymbol{x}_{K}+s\left(\boldsymbol{y}-\boldsymbol{x}_{K}\right)\right)\right|^{p} d_{K, \sigma} s^{d-1} \mathrm{~d} s \mathrm{~d} \gamma(\boldsymbol{y}) .
$$

For a given $\boldsymbol{y} \in \sigma, s \mapsto v\left(\boldsymbol{x}_{K}+s\left(\boldsymbol{y}-\boldsymbol{x}_{K}\right)\right)$ is a polynomial with respect to $s$ with degree lower or equal to $n$, that we write under the form $v\left(\boldsymbol{x}_{K}+s\left(\boldsymbol{y}-\boldsymbol{x}_{K}\right)\right)=\sum_{i=0}^{n} a_{i}(\boldsymbol{y}) s^{i}$. We then use the notation introduced in Definition A.2, which provides

$$
\begin{aligned}
\int_{0}^{\beta}\left|\sum_{i=0}^{n} a_{i}(\boldsymbol{y}) s^{i}\right|^{p} s^{d-1} \mathrm{~d} s & =\beta^{d} \int_{0}^{1}\left|\sum_{i=0}^{n} a_{i}(\boldsymbol{y}) \beta^{i} t^{i}\right|^{p} t^{d-1} \mathrm{~d} t \\
& \geq \beta^{d} C_{p, n} \sum_{i=0}^{n}\left|a_{i}(\boldsymbol{y}) \beta^{i}\right|^{p} \geq \beta^{d+p n} C_{p, n} \sum_{i=0}^{n}\left|a_{i}(\boldsymbol{y})\right|^{p} .
\end{aligned}
$$

This leads to

$$
\int_{D_{K, \sigma}^{(\beta)}}|v(\boldsymbol{x})|^{p} \mathrm{~d} \boldsymbol{x} \geq \beta^{d+p n} C_{p, n} \int_{\sigma} d_{K, \sigma}\left(\sum_{i=0}^{n}\left|a_{i}(\boldsymbol{y})\right|^{p}\right) \mathrm{d} \gamma(\boldsymbol{y})
$$

On the other hand, we have

$$
\begin{array}{r}
\int_{D_{K, \sigma}}|v(\boldsymbol{x})|^{p} \mathrm{~d} \boldsymbol{x}=\int_{\sigma} \int_{0}^{1}\left|v\left(\boldsymbol{x}_{K}+s\left(\boldsymbol{y}-\boldsymbol{x}_{K}\right)\right)\right|^{p} d_{K, \sigma} s^{d-1} \mathrm{~d} s \mathrm{~d} \gamma(\boldsymbol{y}) \\
=\int_{\sigma} \int_{0}^{1}\left|\sum_{i=0}^{n} a_{i}(\boldsymbol{y}) s^{i}\right|^{p} d_{K, \sigma} s^{d-1} \mathrm{~d} s \mathrm{~d} \gamma(\boldsymbol{y}) \\
\leq(n+1)^{p-1} \int_{\sigma}\left(\sum_{i=0}^{n}\left|a_{i}(\boldsymbol{y})\right|^{p}\right) d_{K, \sigma} \mathrm{d} \gamma(\boldsymbol{y}) .
\end{array}
$$

Hence, gathering the above relations, the lemma is proved.

Lemma A.4: Let $n \in \mathbb{N}$ be given. Let $\mathfrak{T}$ be a polytopal mesh in the sense of Definition 3.1. Then, for all $q \in[1,+\infty)$, there holds

$$
\forall v \in \mathbb{P}_{n}\left(\mathbb{R}^{d}\right), \forall K \in \mathcal{M}, \forall \sigma \in \mathcal{F}_{K}, d_{K, \sigma} \int_{\sigma}|v(\boldsymbol{y})|^{q} \mathrm{~d} \gamma(\boldsymbol{y}) \leq \frac{(n+1)^{q-1}}{C_{q, n}} \int_{D_{K, \sigma}}|v(\boldsymbol{x})|^{q} \mathrm{~d} \boldsymbol{x},
$$

where $C_{q, n}$ is defined in Definition A.2 with $p=q$.

Proof. Let $K \in \mathcal{M}, \sigma \in \mathcal{F}_{K}$ and $v \in \mathbb{P}_{n}\left(\mathbb{R}^{d}\right)$ be given. For a given $\boldsymbol{y} \in \sigma, s \mapsto v\left(\boldsymbol{x}_{K}+s\left(\boldsymbol{y}-\boldsymbol{x}_{K}\right)\right)$ is a polynomial with respect to $s$ with degree lower or equal to $n$, that we write under the form $v\left(\boldsymbol{x}_{K}+s(\boldsymbol{y}-\right.$ $\left.\left.\boldsymbol{x}_{K}\right)\right)=\sum_{i=0}^{n} a_{i}(\boldsymbol{y}) s^{i}$. We then use the constant introduced in Definition A.2, which provides

$$
\int_{0}^{1}\left|\sum_{i=0}^{n} a_{i}(\boldsymbol{y}) s^{i}\right|^{q} s^{d-1} \mathrm{~d} s \geq C_{q, n} \sum_{i=0}^{n}\left|a_{i}(\boldsymbol{y})\right|^{q}
$$


and therefore

$$
\int_{D_{K, \sigma}}|v(\boldsymbol{x})|^{q} \mathrm{~d} \boldsymbol{x}=d_{K, \sigma} \int_{\sigma} \int_{0}^{1}\left|\sum_{i=0}^{n} a_{i}(\boldsymbol{y}) s^{i}\right|^{q} s^{d-1} \mathrm{~d} s \mathrm{~d} \gamma(\boldsymbol{y}) \geq C_{q, n} d_{K, \sigma} \int_{\sigma}\left(\sum_{i=0}^{n}\left|a_{i}(\boldsymbol{y})\right|^{q}\right) \mathrm{d} \gamma(\boldsymbol{y}) .
$$

Remarking that $\boldsymbol{y}=\boldsymbol{x}_{K}+s\left(\boldsymbol{y}-\boldsymbol{x}_{K}\right)$ for $s=1$, we have

$$
\int_{\sigma}|v(\boldsymbol{y})|^{q} \mathrm{~d} \gamma(\boldsymbol{y})=\int_{\sigma}\left|\sum_{i=0}^{n} a_{i}(\boldsymbol{y})\right|^{q} \mathrm{~d} \gamma(\boldsymbol{y}) \leq(n+1)^{q-1} \int_{\sigma}\left(\sum_{i=0}^{n}\left|a_{i}(\boldsymbol{y})\right|^{q}\right) \mathrm{d} \gamma(\boldsymbol{y}) .
$$

Gathering the two previous inequalities gives (59).

Since our geometric hypotheses are different from those done in $[7,8]$, we now provide the Sobolev inequalities suited to Definition 3.1 of polytopal meshes. The techniques of proof are identical to that of [8, Theorem 5.3].

Lemma A.5 (Comparison of DG norms): Let $\mathcal{D}$ be a DGGD in the sense of Definition 3.2. Then, for $1 \leq$ $p \leq q<+\infty$, there holds

$$
\forall v \in X_{\mathcal{D}, 0},\|v\|_{\mathrm{DG}, p} \leq((d+1)|\Omega|)^{\frac{q-p}{p q}}\|v\|_{\mathrm{DG}, q} .
$$

Proof. We have

$$
\|v\|_{\mathrm{DG}, p}^{p}=\sum_{K \in \mathcal{M}}\left(\int_{K}\left|\nabla_{\bar{K}} v(\boldsymbol{x})\right|^{p} \mathrm{~d} \boldsymbol{x}+\sum_{\sigma \in \mathcal{F}_{K}} \frac{1}{d_{K, \sigma}^{p-1}} \int_{\sigma}\left|[v]_{K, \sigma}(\boldsymbol{y})\right|^{p} \mathrm{~d} \gamma(\boldsymbol{y})\right) .
$$

Thanks to Young's inequality, we have

$$
\begin{aligned}
\|v\|_{\mathrm{DG}, p}^{p} \leq & \left(\sum_{K \in \mathcal{M}}\left(\int_{K}\left|\nabla_{\bar{K}} v(\boldsymbol{x})\right|^{q} \mathrm{~d} \boldsymbol{x}+\sum_{\sigma \in \mathcal{F}_{K}} \frac{1}{d_{K, \sigma}^{q-1}} \int_{\sigma}\left|[v]_{K, \sigma}(\boldsymbol{y})\right|^{q} \mathrm{~d} \gamma(\boldsymbol{y})\right)\right)^{p / q} \\
& \times\left(\sum_{K \in \mathcal{M}}\left(|K|+\sum_{\sigma \in \mathcal{F}_{K}} d_{K, \sigma}|\sigma|\right)\right)^{(q-p) / q}
\end{aligned}
$$

which leads, since $\sum_{\sigma \in \mathcal{F}_{K}} d_{K, \sigma}|\sigma|=d|K|$, to the result.

Lemma A.6 (Comparison of DG norm and BV norm): For $\bar{v} \in L^{1}\left(\mathbb{R}^{d}\right)$, let us define

$$
\|\bar{v}\|_{\mathrm{BV}}=\sum_{i=1}^{d} \sup \left\{\int_{\mathbb{R}^{d}} \bar{v}(\boldsymbol{x}) \partial_{i} \varphi(\boldsymbol{x}) \mathrm{d} \boldsymbol{x}, \varphi \in C_{c}^{1}\left(\mathbb{R}^{d}\right),\|\varphi\|_{\infty} \leq 1\right\} \in\left(\mathbb{R}^{+} \cup\{+\infty\}\right) .
$$

Then, extending $\Pi_{\mathcal{D}} v$ for all $v \in X_{\mathcal{D}, 0}$ by 0 outside $\Omega$, we have

$$
\forall p \in\left[1,+\infty\left[, \forall v \in X_{\mathcal{D}, 0},\left\|\Pi_{\mathcal{D}} v\right\|_{\mathrm{BV}} \leq d((d+1)|\Omega|)^{\frac{p-1}{p}}\|v\|_{\mathrm{DG}, p}\right.\right.
$$

Proof. We have, for all $v \in X_{\mathcal{D}, 0}$, thanks again to (16),

$$
\left\|\Pi_{\mathcal{D}} v\right\|_{\mathrm{BV}} \leq d \sum_{K \in \mathcal{M}}\left(\int_{K}\left|\nabla_{\bar{K}} v(\boldsymbol{x})\right| \mathrm{d} \boldsymbol{x}+\sum_{\sigma \in \mathcal{F}_{K}} \int_{\sigma}\left|[v]_{K, \sigma}(\boldsymbol{y})\right| \mathrm{d} \gamma(\boldsymbol{y})\right) .
$$

Thanks to Young's inequality, we have

$$
\begin{aligned}
\left\|\Pi_{\mathcal{D}} v\right\|_{\mathrm{BV}} \leq & d\left(\sum_{K \in \mathcal{M}}\left(\int_{K}|\nabla \bar{K} v(\boldsymbol{x})|^{p} \mathrm{~d} \boldsymbol{x}+\sum_{\sigma \in \mathcal{F}_{K}} \frac{1}{d_{K, \sigma}^{p-1}} \int_{\sigma}\left|[v]_{K, \sigma}(\boldsymbol{y})\right|^{p} \mathrm{~d} \gamma(\boldsymbol{y})\right)\right)^{1 / p} \\
& \times\left(\sum_{K \in \mathcal{M}}\left(|K|+\sum_{\sigma \in \mathcal{F}_{K}} d_{K, \sigma}|\sigma|\right)\right)^{(p-1) / p}
\end{aligned}
$$


The results follows.

We now state the discrete Sobolev inequalities result.

Lemma A.7 (Discrete Sobolev inequalities): Let $\mathcal{D}$ be a DGGD in the sense of Definition 3.2. Then, for all $q \in\left[1, \frac{p d}{d-p}\right]$ if $1 \leq p<d$ and $q \in[1,+\infty$ [ otherwise, there exists $C$, depending only on $|\Omega|, k, p, q$ and $d$, such that

$$
\forall v \in X_{\mathcal{D}, 0},\left\|\Pi_{\mathcal{D}} v\right\|_{L^{q}(\Omega)} \leq C\|v\|_{\mathrm{DG}, p}
$$

Proof. Let us first assume that $d=1$. Then we have

$$
\forall \bar{v} \in L^{q}(\mathbb{R}),\|\bar{v}\|_{L^{\infty}(\mathbb{R})} \leq\|\bar{v}\|_{\mathrm{BV}}
$$

which provides (62) owing to Lemma A.6.

We now assume that $d \geq 2$.

As in [7], we follow Nirenberg's technique. First remark that, for all $q \in[1, d /(d-1)]$,

$$
\forall \bar{v} \in L^{q}\left(\mathbb{R}^{d}\right),\|\bar{v}\|_{L^{q}\left(\mathbb{R}^{d}\right)} \leq \frac{1}{2 d}\|\bar{v}\|_{\mathrm{BV}} .
$$

This inequality implies, owing to Lemma A.6,

$$
\forall v \in X_{\mathcal{D}, 0},\left\|\Pi_{\mathcal{D}} v\right\|_{L^{q}\left(\mathbb{R}^{d}\right)} \leq \frac{1}{2 d}\left\|\Pi_{\mathcal{D}} v\right\|_{\mathrm{BV}} \leq \frac{1}{2}\|v\|_{\mathrm{DG}, 1} .
$$

We now handle the case $1<p<d$. We define $\alpha=\frac{p(d-1)}{d-p}>1$ and $p^{\star}=\frac{p d}{d-p}$. For $v \in X_{\mathcal{D}, 0}$, we apply (63) to $\left|\Pi_{\mathcal{D}} v\right|^{\alpha}$ and $q=d /(d-1)$. It yields

$$
\left(\int_{\Omega}\left|\Pi_{\mathcal{D}} v(\boldsymbol{x})\right|^{p^{\star}} \mathrm{d} \boldsymbol{x}\right)^{\frac{d-1}{d}} \leq \frac{1}{2 d} \sum_{i=1}^{d}\left(\left.\sum_{K \in \mathcal{M}} \int_{K}\left|\partial_{i}\right| \Pi_{\bar{K}} v(\boldsymbol{x})\right|^{\alpha}\left|\mathrm{d} \boldsymbol{x}+\sum_{\sigma \in \mathcal{F}} \int_{\sigma}\right|\left[|v|^{\alpha}\right]_{\sigma}(\boldsymbol{y}) \mid \mathrm{d} \gamma(\boldsymbol{y})\right)
$$

denoting by $\left|[w]_{\sigma}(\boldsymbol{y})\right|$ the absolute value of the jump of $w$ at $\boldsymbol{y} \in \sigma$. We observe that, for a.e. $\boldsymbol{x} \in K$, we have $\left.\left.\left|\partial_{i}\right| \Pi_{\bar{K}} v(\boldsymbol{x})\right|^{\alpha}|=\alpha| \Pi_{\bar{K}} v(\boldsymbol{x})\right|^{\alpha-1}\left|\partial_{i} \Pi_{\bar{K}} v(\boldsymbol{x})\right| \leq \alpha\left|\Pi_{\bar{K}} v(\boldsymbol{x})\right|^{\alpha-1}\left|\nabla_{\bar{K}} v(\boldsymbol{x})\right|$. On the other hand, we have, for $\mathcal{M}_{\sigma}=\{K, L\},\left|\left[|v|^{\alpha}\right]_{\sigma}(\boldsymbol{y})\right| \leq 2 \alpha\left(\left|\Pi_{\bar{K}} v(\boldsymbol{y})\right|^{\alpha-1}+\left|\Pi_{\bar{L}} v(\boldsymbol{y})\right|^{\alpha-1}\right) \frac{\left|\Pi_{\bar{K}} v(\boldsymbol{y})-\Pi_{\bar{L}} v(\boldsymbol{y})\right|}{2}$, and, for $\mathcal{M}_{\sigma}=\{K\}$, $\left|\left[|v|^{\alpha}\right]_{\sigma}(\boldsymbol{y})\right| \leq \alpha\left|\Pi_{\bar{K}} v(\boldsymbol{y})\right|^{\alpha-1}\left|\Pi_{\bar{K}} v(\boldsymbol{y})-0\right|$. We then have, using notation (16),

$$
\left(\int_{\Omega}\left|\Pi_{\mathcal{D}} v(\boldsymbol{x})\right|^{p^{\star}} \mathrm{d} \boldsymbol{x}\right)^{\frac{d-1}{d}} \leq \alpha \sum_{K \in \mathcal{M}}\left(\int_{K}\left|\Pi_{\bar{K}} v(\boldsymbol{x})\right|^{\alpha-1}\left|\nabla_{\bar{K}} v(\boldsymbol{x})\right| \mathrm{d} \boldsymbol{x}+\sum_{\sigma \in \mathcal{F}_{K}} \int_{\sigma}\left|\Pi_{\bar{K}} v(\boldsymbol{y})\right|^{\alpha-1}\left|[v]_{K, \sigma}(\boldsymbol{y})\right| \mathrm{d} \gamma(\boldsymbol{y})\right) .
$$

Hence we get, from Young's inequality, since $(\alpha-1) p^{\prime}=p^{\star}$ with $p^{\prime}=p /(p-1)$,

$$
\left(\int_{\Omega}\left|\Pi_{\mathcal{D}} v(\boldsymbol{x})\right|^{p^{\star}} \mathrm{d} \boldsymbol{x}\right)^{\frac{d-1}{d}} \leq \alpha\left(T_{1}\right)^{1 / p}\left(T_{2}\right)^{(p-1) / p},
$$

with

$$
T_{1}=\sum_{K \in \mathcal{M}}\left(\int_{K}\left|\nabla_{\bar{K}} v(\boldsymbol{x})\right|^{p} \mathrm{~d} \boldsymbol{x}+\sum_{\sigma \in \mathcal{F}_{K}} \int_{\sigma} \frac{\left|[v]_{K, \sigma}(\boldsymbol{y})\right|^{p}}{d_{K, \sigma}^{p-1}} \mathrm{~d} \gamma(\boldsymbol{y})\right)=\|v\|_{\mathrm{DG}, p}^{p},
$$

and

$$
T_{2}=\sum_{K \in \mathcal{M}}\left(\int_{K}\left|\Pi_{\bar{K}} v(\boldsymbol{x})\right|^{p^{\star}} \mathrm{d} \boldsymbol{x}+\sum_{\sigma \in \mathcal{F}_{K}} d_{K, \sigma} \int_{\sigma}\left|\Pi_{\bar{K}} v(\boldsymbol{y})\right|^{p^{\star}} \mathrm{d} \gamma(\boldsymbol{y})\right) .
$$

Let us now observe that

$$
\int_{K}\left|\Pi_{\bar{K}} v(\boldsymbol{x})\right|^{p^{\star}} \mathrm{d} \boldsymbol{x}=\sum_{\sigma \in \mathcal{F}_{K}} \int_{D_{K, \sigma}}\left|\Pi_{\bar{K}} v(\boldsymbol{x})\right|^{p^{\star}} \mathrm{d} \boldsymbol{x} .
$$


We write, as in the proof of Lemma A.3,

$$
\int_{D_{K, \sigma}}\left|\Pi_{\bar{K}} v(\boldsymbol{x})\right|^{p^{\star}} \mathrm{d} \boldsymbol{x}=d_{K, \sigma} \int_{\sigma} \int_{0}^{1}\left|\Pi_{\bar{K}} v\left(\boldsymbol{x}_{K}+s\left(\boldsymbol{y}-\boldsymbol{x}_{K}\right)\right)\right|^{p^{\star}} s^{d-1} \mathrm{~d} s \mathrm{~d} \gamma(\boldsymbol{y}) .
$$

We then apply Lemma A.4, letting $n=k$ and replacing $v$ by $\Pi_{\bar{K}} v$. From (59), we get

$$
d_{K, \sigma} \int_{\sigma}\left|\Pi_{\bar{K}} v(\boldsymbol{y})\right|^{p^{\star}} \mathrm{d} \gamma(\boldsymbol{y}) \leq \frac{(k+1)^{p^{\star}-1}}{C_{p^{\star}, k}} \int_{D_{K, \sigma}}\left|\Pi_{\bar{K}} v(\boldsymbol{x})\right|^{p^{\star}} \mathrm{d} \boldsymbol{x},
$$

and concludes the proof that

$$
T_{2} \leq\left(1+\frac{(k+1)^{p^{\star}-1}}{C_{p^{\star}, k}}\right) \int_{\Omega}\left|\Pi_{\mathcal{D}} v(\boldsymbol{x})\right|^{p^{\star}} \mathrm{d} \boldsymbol{x} .
$$

Hence we conclude (62) for $1<p<d$.

Let us finally consider the case $d \leq p$. We select any real value $q_{1}>p$, and we set $p_{1}=d q_{1} /\left(d+q_{1}\right)$. Then we have $1<p_{1}<d \leq p$ and $p_{1}^{\star}=q_{1}$. We apply the result proved for $1<p<d$ above, which yields (62), replacing $p$ by $p_{1}$ and $p_{1}^{\star}$ by $q_{1}$. We then apply Lemma A.5, which allows to bound $\|v\|_{D G, p_{1}}$ by $\|v\|_{D G, p}$.

\section{References}

[1] D. N. Arnold. An interior penalty finite element method with discontinuous elements. SIAM journal on numerical analysis, 19(4):742-760, 1982.

[2] D. N. Arnold, F. Brezzi, B. Cockburn, and L. D. Marini. Unified analysis of discontinuous Galerkin methods for elliptic problems. SIAM J. Numer. Anal., 39(5):1749-1779, 2001/02.

[3] S. C. Brenner and L. Owens. A weakly over-penalized non-symmetric interior penalty method. JNAIAM J. Numer. Anal. Ind. Appl. Math., 2(1-2):35-48, 2007.

[4] E. Burman and A. Ern. Discontinuous Galerkin approximation with discrete variational principle for the nonlinear Laplacian. C. R. Math. Acad. Sci. Paris, 346(17-18):1013-1016, 2008.

[5] E. Burman and P. Zunino. A domain decomposition method for partial differential equations with non-negative form based on interior penalties. SIAM J. Numer. Anal, 44(4):1612-1638, 2006.

[6] D. A. Di Pietro and J. Droniou. A Hybrid High-Order method for Leray-Lions elliptic equations on general meshes. Math. Comp., 86(307):2159-2191, 2017.

[7] D. A. Di Pietro and A. Ern. Discrete functional analysis tools for discontinuous Galerkin methods with application to the incompressible Navier-Stokes equations. Math. Comp., 79(271):1303-1330, 2010 .

[8] D. A. Di Pietro and A. Ern. Mathematical aspects of discontinuous Galerkin methods, volume 69 of Mathématiques \& Applications (Berlin) [Mathematics \& Applications]. Springer, Heidelberg, 2012.

[9] J. Droniou, R. Eymard, T. Gallouët, C. Guichard, and R. Herbin. The gradient discretisation method. working paper or preprint, https://hal.archives-ouvertes.fr/hal-01382358, Nov. 2016.

[10] Y. Epshteyn and B. Rivière. Estimation of penalty parameters for symmetric interior penalty Galerkin methods. J. Comput. Appl. Math., 206(2):843-872, 2007.

[11] A. Ern, A. F. Stephansen, and P. Zunino. A discontinuous Galerkin method with weighted averages for advection-diffusion equations with locally small and anisotropic diffusivity. IMA Journal of Numerical Analysis, 29(2):235-256, 2008.

[12] R. Eymard, C. Guichard, and R. Herbin. Small-stencil 3d schemes for diffusive flows in porous media. M2AN, 46:265-290, 2012.

[13] P. Hansbo and M. G. Larson. Discontinuous Galerkin and the Crouzeix-Raviart element: application to elasticity. M2AN Math. Model. Numer. Anal., 37(1):63-72, 2003. 
[14] R. Herbin and F. Hubert. Benchmark on discretization schemes for anisotropic diffusion problems on general grids. In Finite volumes for complex applications $V$, pages 659-692. ISTE, London, 2008.

[15] L. John, M. Neilan, and I. Smears. Stable discontinuous Galerkin FEM without penalty parameters. In Numerical Mathematics and Advanced Applications ENUMATH 2015, pages 165-173. Springer, 2016. 